\title{
Optimisation of Productivity and Thermodynamic Performance of Metabolic Pathways
}

\author{
Mian Xu, Robin Smith and Jhuma Sadhukhan*
}

Centre for Process Integration, School of Chemical Engineering \& Analytical Science, The University of

$$
\text { Manchester, P.O. Box 88, Manchest er, M60 1QD, United Kingdom }
$$

Keywords: metabolism optimisation, Gibbs free energy change, thermodynamic analysis, pathway analysis, flux balance analysis

\begin{abstract}
In this contribution, a novel optimisation strategy has been presented that combines the metabolic flux analysis and pathway identification with the thermodynamic analysis of cellular metabolic systems. First, an optimal metabolic flux distribution among elementary pathways is identified by LP optimisation subject to constraints on flux balance analysis, pathway analysis and negative Gibbs free energy change for pathways, for achieving the maximum yield of products. The Gibbs free energy change for pathways is calculated from the new transformed Gibbs free energy of formation of external metabolites and cofactors that are in stoichiometric balance in metabolic pathways. The consideration of thermodynamic constraints on pathways ensures the selection of feasible pathways. Thereafter, the Gibbs free energy change of pathways is minimised to predict the optimal reaction conditions that facilitate such pathways. Thus the optimisation approach derives the optimal pathway distribution and conditions for the best performance of cellular systems. The effectiveness of the methodology is demonstrated by a case study on the optimisation of pentose phosphate pathway (PPP) and the glycolysis cycle of the insilico Escherichia coli.
\end{abstract}

\footnotetext{
* Author/s to whom correspondence should be addressed:

E-mail: Jhuma.Sadhukhan@manchester.ac.uk, Ph: +44 161306 4396, Fax: +44 1612367439
} 


\section{Introduction}

Metabolic pathways are complex networks of enzyme catalyzed reaction steps presented in terms of internal metabolites, external metabolites and cofactors (a glossary on the terminologies has been provided in Appendix A). Such pathways, also known as elementary pathways are central to cellular biochemical activities, which channel substrate metabolites into production of energy currency, building blocks for biosynthesis, energy reserves, eliminating waste products, and for recycling reducing equivalents ${ }^{1}$. In this light, to quantify intracellular reaction steps and pathways and infer the objectives of cellular metabolic systems, rational modeling strategies need to be developed for altering or optimising cell properties. Optimisation of cellular properties can achieve physiological endpoints corresponding to the production of a desired external metabolite. Hence, optimal metabolic pathways responsible for the productivity of a desired metabolite can be predicted. A rational modeling approach, such as, based on thermodynamic analysis for cellular properties may also elucidate the spontaneity and existence of driving force for the occurrence of metabolic pathways responsible for a desired product. Thus, the selection of optimal metabolic pathways for the productivity of a desired cellular product based on thermodynamic analysis is driven by the relative degree of spontaneity or feasibility among competing pathways. Furthermore, representation of thermodynamic properties for metabolic pathways in terms of cellular reaction conditions attains the optimal conditions corresponding to the productivity of a desired metabolite. In this work, we have proposed an optimisation based methodology for the productivity and reaction conditions of metabolic pathways using thermodynamic analysis.

In recent years, several theoretical approaches have been developed to assign metabolic priorities through engineered cells. An optimisation-based framework, called ObjFind, has been established to infer and test hypothesized metabolic pathways and objectives depending upon experimental results ${ }^{2}$. A representative 
modeling framework for metabolic analysis is the flux balance analysis (FBA), which can be used to infer the objectives of cellular metabolism ${ }^{3,4,5}$. FBA is a constrained optimisation approach based on linear programming (LP), and provides a desired physiological endpoint, e.g., the maximum growth rate, and its corresponding flux distribution under some culture conditions. In FBA, individual reaction steps and their corresponding metabolic flux balances, rather than metabolic pathways, are considered. The application of FBA has been effectively dealt with metabolic flux analysis problems for various kinds of networks ${ }^{6,7,8}$. Mahadevan et al. ${ }^{9}$ have extended FBA for analyzing the dynamic reprogramming of a metabolic network. This dynamic FBA can be used to understand the dynamic behaviour of metabolic networks, and additionally, provide strategies for the design of a network with a desired objective for metabolic engineering. However, some critical issues about FBA remain unresolved ${ }^{10}$, such as the uniqueness of flux distribution and its practical application. Firstly, the implementation of LP in FBA frequently leads to multiple (or alternate) optima depending on initial starting point. Thus for a same set of enzymes, different metabolic fluxes (that may belong to different pathways) are selected depending on an initial guess on metabolic fluxes. In practice, a set of enzymes is selectively responsible for a pathway or a set of pathways and an alteration of which disrupts such pathways to occur ${ }^{11}$. In addition, it is difficult to apply FBA directly to predict the metabolic genotype-phenotype relation (e.g. gene to cellular expression) from a reaction-based perspective ${ }^{12}$. Hence, FBA, without consideration of pathways, does not capture the genetic or enzymatic manipulation of cellular activities. Pathway analysis, on the other hand, is aimed at genetic / enzymatic manipulation of cells, which enables any steady-state metabolic network to be expressed as a collection of elementary pathways. Each elementary pathway is stoichiometrically feasible for a minimum set of enzymes ${ }^{13}$, and the activity of which can be controlled by inhibition or activation of its responsible enzymes. All these elementary pathways should be regarded as the true functional units of metabolic systems consisting of a series of irreversible reaction steps. Thus, a cell can be regulated by selective control of activities of elementary pathways to enhance the yield and rate of a metabolic production. Hence, we combine pathway analysis with FBA to integrate metabolic engineering to genetic technology for the 
synthesis of novel products or redirecting metabolite fluxes towards a desired product. This also eliminates the existence of infeasible flux distribution caused by FBA uncertainty.

Optimal metabolic fluxes based on mass balance thus obtained by combining pathway and flux balance analyses does not ensure feasibility of pathways. Hence, thermodynamic analysis can be instrumental to the selection of feasible pathways and identifying optimal cellular environment for metabolic systems. Thermodynamic insights into metabolic reaction networks or pathways are useful in estimating the key parameters in biotechnological cultures and thus to address reaction viability of bioprocesses ${ }^{14}$. In thermodynamic terms, the difference in Gibbs free energy sets the driving force for any system undergoing changes. For any phenomenon occurring spontaneously, its Gibbs free energy change ought to be negative. This is the basis for assessing the thermodynamic feasibility of a pathway in metabolic systems. Moreover, a pathway for which the free energy change is large and negative has an equilibrium that favors the side of products. Recently, Gibbs free energy changes for individual reaction steps in a metabolic pathway have been presented by Nolan et al. ${ }^{1}$ in order to consider negative constraint on Gibbs free energy change for individual metabolic pathways in pathway analysis. In their study, the Gibbs free energies of formation of metabolic species are estimated using group contribution theory ${ }^{15}$. They have applied directionality criterion for net mss flux in the form of negative Gibbs free energy change to a pathway, as opposed to a reaction step. In many cases, cellular biochemistry, through multi-functional enzyme action, substrate channeling, or other mechanisms, couples an energetically unfavorable metabolic reaction to an energetically favorable one. In these cases, the negative Gibbs free energy change criterion applied to individual reactions would incorrectly predict opposing flux directions for consecutive reactions. Nolan et al. ${ }^{1}$ presented an example of glycolysis which includes at least three reaction steps with unfavorable Gibbs free energy changes in the established direction from glucose to lactate ${ }^{16}$.

In this paper, we have introduced the new transformed Gibbs free energies of formation ${ }^{17}$ in terms of $p H$ 
and ionic strength, which are more suitable for biochemical reaction systems, for external metabolites and cofactors that are stoichiometrcally balanced in individual elementary pathways. Hence, in our work, Gibbs free energy changes have been directly presented for elementary pathways in a metabolic system as opposed to the work by Nolan et al. ${ }^{1}$, who considered thermodynamic descriptors for reaction steps to predict that for pathways. Moreover, Gibbs free energy changes of pathways have been minimised to attain the optimal pH and ionic strength conditions to facilitate such pathways.

The paper is structured as follows. The methodology ${ }^{18}$ for the elucidation and optimisation of metabolic systems has been detailed in the first part. In the second part, a case study on the metabolism network of pentose phosphate pathways (PPP) and glycolysis cycle of in silico Escherichia coli has been established to demonstrate the effectiveness of this methodology.

\section{Methodology}

\subsection{Thermodynamic analysis of metabolic reaction network}

Thermodynamic feasibility and optimisation of metabolic pathways based on Gibbs free energy changes is presented to formulate the optimisation problem for metabolic productivity and optimal reaction conditions. Generally speaking, there are two kinds of reaction equations, chemical equations which balance elements and charge, and biochemical equations written in terms of biochemical reactants at a specified $p H^{17}$. In biochemical equations, hydrogen numbers are assumed fixed at a constant $p H$. The conventional thermodynamic properties thus can not represent biochemical systems precisely. Therefore, it is necessary to define the new transformed thermodynamic properties, like Gibbs free energy of formation, for biochemical reactants ${ }^{17}$. Metabolic pathways can be expressed by biochemical equations. Hence, the new transformed Gibbs free energies of formation should be computed for the metabolites in a system. The 
calculation of the standard formation Gibbs free energy of biochemical reactants as a function of $p H$ and an ionic strength $I$ is illustrated in Fig. 1.

\subsubsection{Gibbs free energy change for biochemical reaction}

The Gibbs free energy $G$ for a reaction system at a specific temperature $(T)$ and pressure $(P)$ is described in terms of species.

$$
G=\sum_{i=1}^{N} n_{i} \mu_{i}
$$

In Eq. (1), $\mu_{i}$ and $n_{i}$ are chemical potential and amount of specie $i$ respectively and $N$ is the number of species in the system.

Eq. (1) provides the basis for deriving the data on the Standard formation Gibbs free energy $\Delta G_{f}{ }^{o}$ of individual species. The superscript ${ }^{\circ}$ and the word standard mean that the species are in aqueous solutions at $1 \mathrm{M}(1 \mathrm{M}=1 \mathrm{~mol} / \mathrm{l})$, while the interactions between ions and water are same as that in infinite dilution. Most of the $\Delta G_{f}{ }^{o}$ values are taken from the NBS Tables of Chemical Thermodynamic Properties ${ }^{19}$. The $\Delta G_{f}{ }^{o} \quad$ values for the ATP series are from ${ }^{20}$.

The thermodynamic properties of a solution are affected by the interactions between the species in it. Therefore, the effect of ionic strength in a solution plays an important role in determining its thermodynamic state. Since the biochemical reactions mostly work at ionic strength which ranges from 0.1 to 0.3 ( $I=0.1-0.3 M)$, the effects of the solution ionic strength have to be considered for the correction of the standard formation Gibbs free energy values. The standard formation Gibbs free energy of specie $i$ at an ionic strength $I$ can be calculated from the one at zero ionic strength using Eq. (2) ${ }^{21}$. 
$\Delta G_{f, i}^{o}=\Delta G_{f, i}^{o}(I=0)-2.91842 z_{i}^{2} I^{1 / 2} /\left(1+B I^{1 / 2}\right)$

The formation Gibbs free energy is expressed in $k J / m o l, \quad z_{i}$ is the charge in specie $i$, and $B=1.6 L^{1 / 2} \mathrm{~mol}^{1 / 2}$.

Biochemical reactions are enzyme-catalysed reactions at a specified $p H$. Many of the reactants are weak acids so that $\mathrm{H}^{+}$is also a reacting specie. When the concentration of a reacting specie is specified at an equilibrium, the thermodynamic properties of the solution depend on its specific concentration, as well as on the temperature and pressure. Thus, a new standard formation Gibbs free energy for species in a biochemical reaction is introduced as a function of hydrogen ion concentration (Eq. (3)).

$$
\begin{aligned}
\Delta G_{f, i}^{\prime o} & =\Delta G_{f, i}^{o}-N_{i}(H)\left\{\Delta G_{f}^{o}\left(H^{+}\right)+R T \ln \left(\left[H^{+}\right] / c^{o}\right)\right\} \\
& =\Delta G_{f, i}^{o}-N_{i}(H)\left\{\Delta G_{f}^{o}\left(H^{+}\right)-2.303 R T \times p H\right\}
\end{aligned}
$$

In Eq. (3), $N_{i}(H)$ is the number of hydrogen atoms in species $i$, and $p H=-\log \left(\left[H^{+}\right] / c^{o}\right)$

In biochemical reactions, the reactants consist of species in equilibrium. Once we have calculated the standard formation Gibbs free energy $\Delta G_{f, i}^{\prime}{ }^{o}$ at a $p H$ for all the species of a reactant, the next step is to combine the $\Delta G_{f, i}^{\prime}{ }^{o}$ values of these species as an integrated property for the reactant. The standard formation Gibbs free energy $\Delta G_{f}^{\prime}$ of reactants at a specific $p H$ is given by Eq. (4). Since all the external metabolites and cofactors in metabolism networks are expressed as biochemical reactants, thus, their corresponding formation Gibbs free energy $\Delta G_{f, e \in E M}^{\prime}$ should also calculated by Eq. (4).

$$
\Delta G_{f, e \in E M}^{\prime}=\Delta G_{f}^{\prime}(\text { react })=-R T \ln \left\{\sum_{i=1}^{N_{\text {isq }}} \exp \left(-\Delta G_{f, i}^{\prime o} / R T\right)\right\}
$$

In Eq. (4) $N_{\text {iso }}$ is the number of species of the reactant, and $\Delta G_{f, i}^{\prime}{ }^{\circ}$ is the standard formation Gibbs free energy of species of a reactant, $\Delta G_{f}^{\prime}($ react $)$. 
From Eqs. (2), (3) and (4), the standard formation Gibbs free energies of the external metabolites and cofactors, $\Delta G_{f, e \in E M}^{\prime}$, in a metabolic pathway can be evaluated at a given $p H$ and ionic strength. In the following section, an illustration on the calculation of the standard formation Gibbs free energy of metabolites is presented.

\subsubsection{An example for calculation}

An example is presented herein to elucidate the procedure of formation Gibbs free energy calculation of biochemical reactants. Reactant ATP in biochemical reactions refers to an equilibrium mixture of ATP $^{4-}$, HATP $^{3-}$ and $H_{2} A T P^{2-}$ at a specified $p H$. The standard formation Gibbs free energy for ATP ${ }^{4-}$, HATP ${ }^{3-}$ and $\mathrm{H}_{2} \mathrm{ATP}^{2-}$ at $25^{\circ} \mathrm{C}, 1 \mathrm{bar}$, and zero ionic strength $(I=0)$, as well as their ionic charges and hydrogen atom numbers are shown in Table 1.

Firstly, the formation Gibbs free energy of species $A T P^{4-}, H_{A T P^{3-}}$ and $H_{2} A T P^{2-}$ at $I=0.28$ are calculated using Eq. (2).

$$
\begin{aligned}
\Delta G_{f, A T P^{4-}}{ }^{o}(I=0.28) & =\Delta G_{f, A T P^{4-}}^{o}(I=0)-2.91842 z_{A T P^{4-}}{ }^{2} I^{1 / 2} /\left(1+B I^{1 / 2}\right) \\
& =-2573.49-2.91842 \times 4^{2} \times 0.28^{1 / 2} /\left(1+1.6 \times 0.28^{1 / 2}\right)=-2586.87 \mathrm{~kJ} / \mathrm{mol} \\
\Delta G_{f, \mathrm{HATP}^{3-}}{ }^{o}(I=0.28) & =\Delta G_{f, \mathrm{HATP}^{3-}}^{o}(I=0)-2.91842 \mathrm{z}_{\mathrm{HATP}^{3-}}{ }^{2} I^{1 / 2} /\left(1+B I^{1 / 2}\right) \\
= & -2616.87-2.91842 \times 3^{2} \times 0.28^{1 / 2} /\left(1+1.6 \times 0.28^{1 / 2}\right)=-2624.40 \mathrm{~kJ} / \mathrm{mol} \\
\Delta G_{f, H_{2} A T P^{-2}}{ }^{o}(I=0.28) & =\Delta G_{f, H_{2} A T P^{2-}}^{o}(I=0)-2.91842 z_{\mathrm{H}_{2} A T P^{2-}}{ }^{2} I^{1 / 2} /\left(1+B I^{1 / 2}\right) \\
= & -2643.58-2.91842 \times 2^{2} \times 0.28^{1 / 2} /\left(1+1.6 \times 0.28^{1 / 2}\right)=-2646.93 \mathrm{~kJ} / \mathrm{mol} \\
\Delta G_{f, H^{+}}{ }^{o}(I=0.28)= & \Delta G_{f, H^{+}}^{o}(I=0)-2.91842 \mathrm{z}_{i}^{2} I^{1 / 2} /\left(1+B I^{1 / 2}\right) \\
= & 0-2.91842 \times 2^{2} \times 0.28^{1 / 2} /\left(1+1.6 \times 0.28^{1 / 2}\right)=-0.84 \mathrm{~kJ} / \mathrm{mol}
\end{aligned}
$$


Next, the formation Gibbs free energy of species at a $p H=6.8$ are calculated by Eq. (3).

$$
\begin{aligned}
\Delta G_{f, A T P^{4-}}^{\prime o} & =\Delta G_{f, A T P^{4-}}{ }^{o}-N_{A T P^{4-}}\left(H^{+}\right)\left\{\Delta G_{f}^{o}\left(H^{+}\right)-2.303 R T \times p H\right\} \\
& =-2586.87-12 \times\{-0.84-2.303 \times 0.008314 \times 298 \times 6.8\}=-2111.24 \mathrm{~kJ} / \mathrm{mol} \\
\Delta G_{H A T P^{3-}}^{\prime o} & =\Delta G_{f, H A T P^{3-}}^{o}-N_{H A T P^{3-}}\left(H^{+}\right)\left\{\Delta G_{f}^{o}\left(H^{+}\right)-2.303 R T \times p H\right\} \\
& =-2624.40-13 \times\{-0.84-2.303 \times 0.008314 \times 298 \times 6.8\}=-2109.13 \mathrm{~kJ} / \mathrm{mol} \\
\Delta G_{f, H_{2} A T P^{2-}}^{\prime o} & =\Delta G_{f, H_{2} A T P^{2-}}^{o}-N_{H_{2} A T P^{2-}}\left(H^{+}\right)\left\{\Delta G^{o}{ }_{f}\left(H^{+}\right)-2.303 R T \times p H\right\} \\
& =-2646.93-14 \times\{-0.84-2.303 \times 0.008314 \times 298 \times 6.8\}=-2092.02 \mathrm{~kJ} / \mathrm{mol}
\end{aligned}
$$

Reactant ATP is a mixture of the three species: $A T P^{4-}, H A T P^{3-}$ and $H_{2} A T P^{2-}$. Thus, the number of species for ATP is $3\left(N_{\text {iso }}=3\right)$. The formation Gibbs free energy of reactant ATP is calculated using Eq. (4).

$$
\begin{aligned}
\Delta G_{f}^{\prime}(A T P) & =-R T \ln \left\{\exp \left(-\Delta G_{f, A T P^{4-}}^{o 0} / R T\right)+\exp \left(-\Delta G_{f, H A T P^{3-}}^{\prime} / R T\right)+\exp \left(-\Delta G_{f, H_{2} A T P^{2-}}^{\prime} / R T\right)\right\} \\
& =-2376.47 \mathrm{~kJ} / \mathrm{mol}
\end{aligned}
$$

\subsection{Flux balance analysis and pathway analysis}

Flux balance analysis (FBA) and pathway analysis are used for the systematic enumeration of elementary pathways and metabolic flux distributions among them.

FBA is used to describe metabolic system models that include a complete list of reactions and metabolites (external as well as internal) and cofactors involved in each reaction step, in a quantitative manner. For FBA of metabolic systems, the information required is the stoichiometry of metabolic reaction steps, mass balance around internal metabolites under pseudo-steady state and the uptake of external metabolite sources $^{22}$. The process of flux balance analysis is illustrated in Fig. 2. 
In FBA, a stoichiometry of metabolic reactions is presented by a $X \times N$ stoichiometric matrix $\bar{S}_{(i, j)}$, in terms of $X$ number of internal metabolites $i \in I M$, in a total of $N$ reactions $j \in R$. Since it is reasonable to place the internal metabolites of a system into a steady state, a set of linear homogeneous equations based on mass balance can be derived. In matrix notation, the expression is presented in Eq. (5). $\bar{V}$ is a $N \times 1$ flux vector and represents the flux distribution of reactions in a metabolic system.

$$
\bar{S} \times \bar{V}=0
$$

Pathway analysis is aimed at genetic / enzymatic manipulation of cells, which enables any steady-state metabolic network to be expressed as a collection of elementary pathways. Each elementary pathway is stoichiometrically feasible for a minimum set of enzymes, and its overall activity can be controlled by inhibition or activation of its responsible enzymes. Therefore, pathway analysis is combined with FBA to eliminate the existence of infeasible flux distribution caused by FBA uncertainty, and also integrate metabolic engineering to genetic technology for achieving the synthesis of novel products or redirecting metabolite fluxes towards a desired product. Based on the combination of FBA and pathway analysis, the optimal mass flux distribution among these elementary pathways for the maximum productivity of a desired metabolite can be predicted. Moreover, each elementary pathway is a collection of individual reaction steps. The overall reaction of an elementary pathway includes only external metabolites and cofactors. Thus, two stoichiometric matrices, $\bar{A}$ and $\bar{U}$, can be derived from pathway analysis. $\bar{A}_{(j, p)}$ represents a $N \times M$ stoichiometric matrix, in terms of $N$ number of reactions $j \in R$, in a total of $M$ elementary pathways $p \in P . \bar{U}_{(p, e)}$ is a $M \times Y$ stoichiometric matrix in terms of $Y$ external metabolites and cofactors $e \in E M$ in a total of $M$ elementary pathways $p \in P . \bar{B}$ is a $M \times 1$ flux vector referring to the flux distribution in elementary pathways $p(p \in P)$ detected in the system, the values of which can not be negative. The relationship between reaction flux distribution $\bar{V}$ and pathway flux distribution $\bar{B}$ can be expressed as: 
If $\overline{V E}$ is a $Y \times 1$ flux vector in terms of $Y$ number of external metabolites $e(e \in E M)$ in a system, the overall equations for elementary pathways give rise to the stoichiometry of overall reactions (Eq. (7)).

$\overline{V E}=\bar{U} \times \bar{B}$

Substituting Eq. (6) into Eq. (5), Eq. (8) is resulted.

$\bar{S} \times(\bar{A} \times \bar{B})=0$

\subsection{Optimisation Approach and Inclusion of Thermodynamic Analysis}

An optimisation approach is developed, which combines the metabolic flux analysis and pathway identification as illustrated by Eqs. (5)-(8) in section 2.2 and the thermodynamic analysis discussed in Eqs. (2)-(4) in section 2.1 (Fig. 3). The optimisation approach is presented as two modules. In module 1, the objective is the productivity of an external metabolite (desired product) and is maximised by linear programming (LP). In module 2, thermodynamic optimisation (NLP) in terms of minimisation of the total Gibbs free energy change of a metabolic system is carried out to predict the optimal cellular conditions. The formulations for module 2 are non-linear due to the calculation of formation Gibbs free energy of external metabolites and cofactors. The detailed algorithm of the optimisation strategy is presented in Fig. B1 in Appendix B, and illustrated in the following sections.

\subsubsection{Productivity Maximisation}

In addition to the existing approaches ${ }^{3,5,13}$, we have combined the flux balance analysis together with pathway analysis (section 2.2; Eqs. (5)-(8)) to achieve the optimal flux distribution among pathway modes, 
rather than for individual reactions, which satisfies the desired physiological endpoint, eg., the maximum productivity of external metabolites. Moreover, an inequality thermodynamic constraint defining the negative Gibbs free energy change for individual metabolic pathways is used to ensure the feasibility of flux balance analysis (Eq. (9)). The new thermodynamic properties designed for biochemical reactants ${ }^{17}$ in terms of the standard formation Gibbs free energy change $\left(\Delta G_{f, \epsilon \in E M}^{\prime}\right)$ are predicted for external metabolites and cofactors in individual metabolic pathways (Eqs. (2), (3)-(4)). Based on these values, the standard pathway Gibbs free energy changes $\left(\Delta G_{p \in P}^{\prime o}\right)$ are determined using stoichiometric balance among external metabolites and cofactors in individual elementary pathways. The constraint for the negative Gibbs free energy change of pathways is expressed in Eq. (9).

$\overline{G P}=\bar{U} \times \overline{G E} \leq 0$

$\overline{G P}$ is a $M \times 1$ vector of the standard Gibbs free energy changes for elementary pathways ( $\left.\Delta G_{p \in P}^{\prime o}\right), \overline{G E}$ is a $Y \times 1$ vector of the standard formation Gibbs free energy of external metabolites and cofactors ( $\Delta G_{f, e \in E M}^{\prime}$ ) at specified $p H$ and ionic strength, and $M$ is the number of elementary pathways included in a system.

The strategy for a productivity maximisation is depicted in Fig. 4. The productivity of a desired external metabolite is defined as the objective function to be maximised for a given set of reacting rates of substrates. The decision variable is the pathway flux distribution $\bar{B}$. The LP problem formulation can be expressed as follows:

Maximise $\overline{O B J E C T}\{e \in O B J\} \quad$ (Productivity Maximisation)

Subject to Eqs. (6), (8), (9) and $\bar{B} \geq 0$

$$
\overline{r_{\text {reacting }}}=\left\{r_{\text {reacting }_{1}}, r_{\text {reacting }_{2}}, \ldots, r_{\text {reacting }_{e}}, \ldots / e \in U P T\right\}
$$


$\overline{r_{\text {reacting }}}$ in Eq. (10) presents the reacting rates of external metabolites ( $\left.e \in U P T\right)$ acting as precursors or substrates (sources) in a system and is user specified or a controlling variable. $\overline{\text { OBJECT }}$ is the property vector of the productivity of a desired external metabolite $e$, which is defined as the objective function to be maximised for a given set of substrates.

\subsubsection{Gibbs free energy minimisation}

From productivity maximisation, the optimal flux distribution of pathways $\left(\bar{B}^{\text {opt }}\right)$ and the maximum generation of the desired product are achieved. The next step is to determine the minimum total Gibbs free energy change of metabolic pathways. Therefore, the optimal flux distribution achieved by the productivity maximisation is used as an input to the thermodynamic evaluation (Eq. (11)). The NLP problem formulation can be expressed as follows:

Minimise $\quad G_{\text {tot }}$

(Gibbs free energy change minimisation)

Subject to: $\quad$ Eqs. (2)-(4), (9), (10)

$$
\begin{aligned}
& \bar{B}=\bar{B}^{o p t} \\
& G_{\text {tot }}=\overline{G P}^{T} \times \bar{B} \\
& \bar{B} \geq 0
\end{aligned}
$$

Negative Gibbs free energy change is also used as a constraint in optimisation. $G_{\text {tot }}$ is the total Gibbs free energy change of the system (Eq. (12)), which is defined as the objective function to be minimised. Since $\overline{G P}^{T}$ is presented as a function of cellular $p H$ and ionic strength (Eqs. (2), (3) and (4)), the minimisation of Gibbs free energy change results into optimal cellular conditions such as $p H$ and ionic strength $I$. Since 
cellular metabolism is a very tightly controlled process, the above two input variables can be optimised within very narrow ranges of limits specified, e.g. $p H=6.8-7$ and $I=0.28-0.3$.

In the context of the overall metabolism engineering design procedure, the proposed methodology (Eqs. (1)-(12) and Figs. 3-5) can be integrated with the experimental program and industrial processes. Its purpose is to systematically screen and set up the incentives for the promising pathways towards a performance target. A priority selection among metabolic elementary pathways can be achieved from flux and energy constrained optimisation analysis of a metabolism system. The differences in thermodynamic driving forces for the selected pathways provide a basis for experiment measurements. The optimal cellular conditions of $p H$ and ionic strength attained by thermodynamic optimisation are valuable for practical experiments or industrial processes. In addition to biological insights, this approach can be adopted in the construction of engineered metabolism pathways by enzyme controlling or some other gene altering technologies. Based on our model, a functional network of elementary metabolic pathways and their predicted flux distribution can be established to govern the cellular metabolic system towards achieving an objective.

\section{Case Study}

A case study on the synthesis of pentose phosphate pathways (PPP) and glycolysis of in silico model of Escherichia coli metabolism has been used to illustrate the proposed optimisation approach.

\subsection{The representation of the metabolic network}

The metabolism network under consideration is embedded with the glycolytic pathway and the pentose phosphate pathway in the in silico model of E. coli metabolism. This network incorporated 26 metabolites 
(4 external metabolites, 15 internal metabolites, 7 cofactors) and 19 metabolic reactions (Tables 2-3). An overview of the reaction scheme for the model is indicated in Fig. 6. Among the external metabolites, glucose-6-phosphate is considered as the only carbon source consumed through the system while producing metabolic products. Other external metabolites include carbon dioxide, Ribose 5-phosphate, and pyruvate. Pyruvate has been assumed as the objective sink for the productivity maximisation.

Formulation of the pathway analysis in a network has been described previously ${ }^{23}$. Thirteen elementary path modes have been derived from computation as shown in Table 4 . Fig. 7 describes the pathway mode 3 as an example to illustrate the reactions involved in this pathway.

Based on the results of flux balance analysis and pathway analysis, a stoichiometry of metabolic reactions is presented by a $15 \times 19$ stoichiometric matrix $\bar{S}_{(i, j)}$ (Table 5), and a $19 \times 13$ stoichiometric matrix $\bar{A}$ is derived from the stoichiometry of reactions in each pathway (Table 6). A $13 \times 11$ stoichiometric matrix $\bar{U}$ presenting the stoichiometry of external metabolites and cofactors in each pathway is shown in Table 7.

\subsection{Thermodynamic properties of the external metabolites and cofactors}

All the biochemical species of the external metabolites and cofactors involved in the metabolism network (Fig. 6) as well as their corresponding thermodynamic properties of the standard formation Gibbs free energy at $25^{\circ} \mathrm{C}, 1$ bar and $\mathrm{I}=0$, are illustrated in Table 4 . Firstly, the standard formation Gibbs free energy of these species, at an initial $p H$ and ionic strength $(p H=6.8, I=0.28)$ is calculated using Eq. (2) and (3) respectively, based on their hydrogen atom numbers $N_{i}\left(H^{+}\right)$and the charge $z_{i}$ (Table 8). Next, the standard formation Gibbs free energy for all the external metabolites and, like ATP, ADP and inorganic phosphate, are calculated using Eq. (4), shown in Table 9. 


\subsection{Optimisation Approach}

\subsubsection{Productivity maximisation}

For the productivity maximisation, the objective is to maximise the product flux of pyruvate using the flux balance analysis (Eqs. (5)-(10)). The reacting rate of glucose-6-phosphate has been specified to be $115 \mathrm{mmol} / \mathrm{gDCWh}{ }^{24}$. Eqs. (6), (9)-(10) are solved using the LP optimisation solver in General Algebraic Modeling System (GAMS)/BDMLP on a Pentium ${ }^{\circledR}$ 4CPU, $3 \mathrm{GHz}$ and $512 \mathrm{MB}$ of RAM. The computational time is 1.7 seconds. It yields the theoretical maximum productivity rate of pyruvate of 115 $\mathrm{mmol} / \mathrm{gDCWh}$. This is obviously maximised compared to the unoptimised pyruvate rate of 44.3 $\mathrm{mmol} / \mathrm{gDCWh}$, from experiments ${ }^{24}$. The corresponding pathways obtained that are responsible for the maximum productivity of pyruvate, are modes 2, 4, 7 and 9 in Fig. 8. To demonstrate the importance of Gibbs free energy change as a sufficient constraint to optimisation, two sets of optimal pathway distributions derived with and without Gibbs free energy constraint is illustrated in Fig. 9. Their corresponding Gibbs free energy changes are summarized in Table 10. The optimal pathway modes derived from optimisation without Gibbs free energy constraint are modes 1, 2, 3, 4, 5, 6, 7, 8, 9, 10, 11 and 12, but some of them have positive Gibbs free energy changes which oppose their thermodynamic feasibility. However, with Gibbs free energy constraint, the corresponding optimal pathways derived are modes 2, 4, 7, and 9, all of which have been strictly constrained to ensure the feasibility and direction of the pathways. The Gibbs free energy change for this process at the initial conditions is $-54696.774 \mathrm{~kJ} \cdot \mathrm{mol}^{-1}$. Additionally, the results in terms of reaction flux distribution and productivity solely from FBA ${ }^{3,4,5}$ are presented in Table 11 in order to establish the distinction of the combined FBA and pathway analysis, introduced in this work. Although the maximum productivity derived from FBA alone is also $115 \mathrm{mmol} / \mathrm{gDCWh}$, which is the same as that obtained from the combined FBA and pathway analysis, the feasibility of this reaction flux distribution is uncertain. Therefore, pathway analysis is still needed further to distribute these reaction 
fluxes into different pathways so as to capture the genetic or enzymatic manipulation of cellular activities. Based on the reaction flux distribution from FBA alone, a set of pathway distributions with the corresponding Gibbs free energy changes is derived as shown in Table 12. The pathways selected from FBA alone are 3 and 6, between which the pathway 3 is an infeasible pathway resulting into positive Gibbs free energy change of $17539.340 \mathrm{~kJ} / \mathrm{mol}$. Thus, we may conclude that only the methodology combining FBA and pathway analysis incorporating thermodynamic constraints can ensure the representation of the metabolic genotype-phenotype relations and related cellular control activities.

\subsubsection{Gibbs free energy minimisation}

To predict the optimal reaction conditions, the optimal flux distribution achieved by the productivity maximisation in section 3.3.1 is used as an input to thermodynamic optimisation. Using Eqs. (2), (3)-(4), the standard formation Gibbs free energy of the external metabolites and cofactors of the system (Fig. 6) as functions of $p H$, ionic strength $I$ is computed. The expression of the standard pathway Gibbs free energy changes for pathway modes are derived from the stoichiometry of the overall reaction equations of these pathways. The objective function is the minimisation of the total Gibbs free energy for all the pathways included (Eq. (13)). The problem is non-linear due to Eqs. (2), (3)-(4). This NLP optimisation problem is solved via the NLP optimisation solver in General Algebraic Modeling System (GAMS)/CONOPT on a Pentium ${ }^{\circledR}$ 4CPU, $3 \mathrm{GHz}$ and $512 \mathrm{MB}$ of RAM. The computational time is 2.3 seconds. The result of the Gibbs free energy minimisation is presented in Table 13. The optimal cellular level reaction conditions obtained are $p H=7$ and $I=0.3$. The corresponding Gibbs free energy change for this process is $-67608.11 \mathrm{~kJ} \cdot \mathrm{mol}^{-1}$, which is significantly minimised compared to the initial value $\left(-54696.774 \mathrm{~kJ} \cdot \mathrm{mol}^{-1}\right)$. Fig. 10 shows that the Gibbs free energy changes for pathway 2, 4 and 9, as well as the total Gibbs free energy change is obviously minimised after minimisation, although the Gibbs fee energy change of mode 7 is slightly increased. 


\section{Conclusion}

A novel optimisation methodology has been presented for the productivity and thermodynamic performance of metabolic systems. The theoretical connection between flux balance analysis and pathway analysis is well established. Their combined application has been integrated with the thermodynamic constraints on Gibbs free energy based driving force in order to predict the maximum productivity of desired products and the optimal metabolic flux distribution. Moreover, thermodynamic optimisation in terms of the Gibbs free minimisation has been successfully developed for metabolic systems, from which, the best cellular conditions are predicted. The heuristic idea of introducing thermodynamic analysis into metabolic engineering presents a new way to rationalize metabolic pathway analysis, hence, providing a better control mechanism for industrial bioprocesses. The work presented in this paper is an essential step forward in establishing cellular pathway level control for productivity maximisation. 


\section{Appendix A}

\section{Glossary}

\section{Metabolism:}

The processes occur within living cells or organisms that are necessary for the maintenance of life. In metabolism some substances are broken down to yield energy for vital processes while other substances, necessary for life, are synthesized.

\section{Metabolites:}

Substances consumed or produced by metabolism, can be external or internal to a cellular system.

\section{External metabolites:}

Metabolites buffered by connection to reservoirs. They can be considered to be sources and sinks (nutrients and waste products, stored or excreted products, or precursors for further transformations), exchanged be tween a cellular system and its environment.

\section{Internal metabolites:}

Metabolites only participate in reactions of the model, the formation of which are exactly balanced by consumption within a cellular system.

\section{Cofactors:}

Substances, such as metallic ions or coenzymes, must be associated with an enzyme for the enzyme to function. 


\section{Appendix B}

\section{Gibbs Free Energy Calculation}

Standard formation Gibbs free energy of external metabolites and cofactors ( $\overline{G_{f}}(e), \forall e \in E M \quad$ )

$$
\downarrow
$$

Set initial operating condition $p H^{o}, I^{\circ}$

Formation Gibbs free energy of external metabolites and cofactors as a function of $\overline{G_{f}}, p H, I$

$$
\overline{G_{f}^{\prime}}=f\left(\overline{G_{f}}, p H, I\right)
$$

Evaluate the Gibbs free energy change for pathways

$$
\overline{G_{r}^{\prime}}=\bar{U} \times \overline{G_{f}^{\prime}}
$$
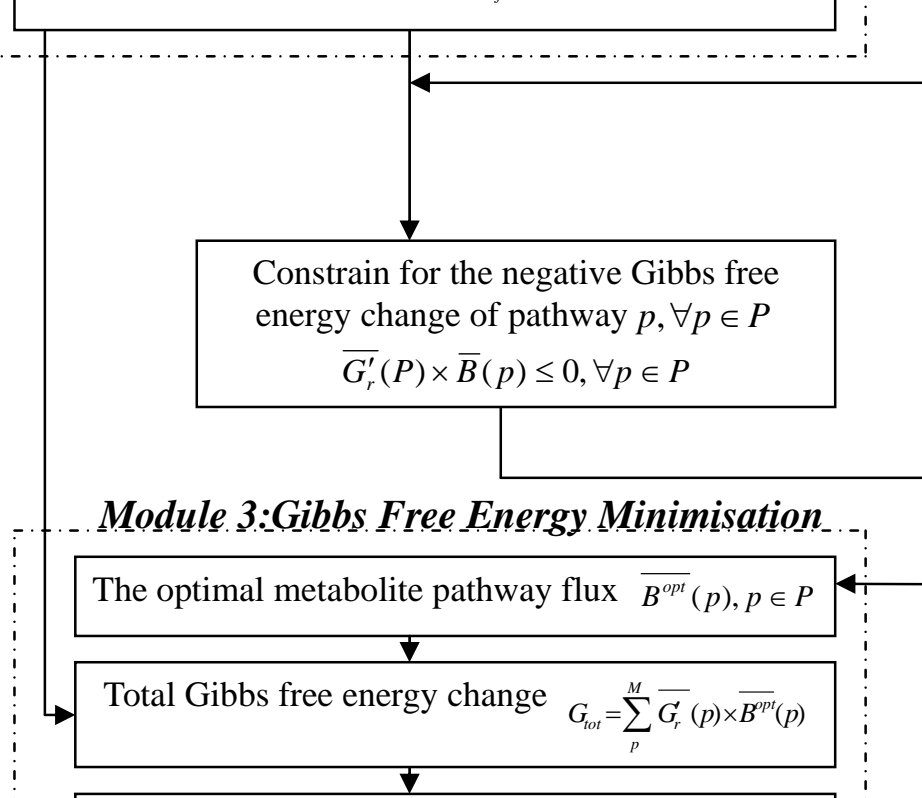

Total Gibbs free energy change minimisation

$$
1
$$

The optimal operating condition

$$
p H_{o p t}, I_{o p t}
$$

\section{Module 2: Productivity Maximisation}

Identify the reactions and their stoichiometry of internal metabolites ( reaction $j, \forall j \in R, \bar{S}(i, j), \forall i \in I M, j \in R$ )

Identify the pathways and their stoichiometry of metabolic reactions ( pathway $p, \forall p \in P, \bar{A}(j, p), \forall j \in R, p \in P$ )

Identify the overall reaction for each pathway and its stoichiometry of external metabolites and cofactors $(\bar{U}(p, e), \forall p \in P, e \in E M)$

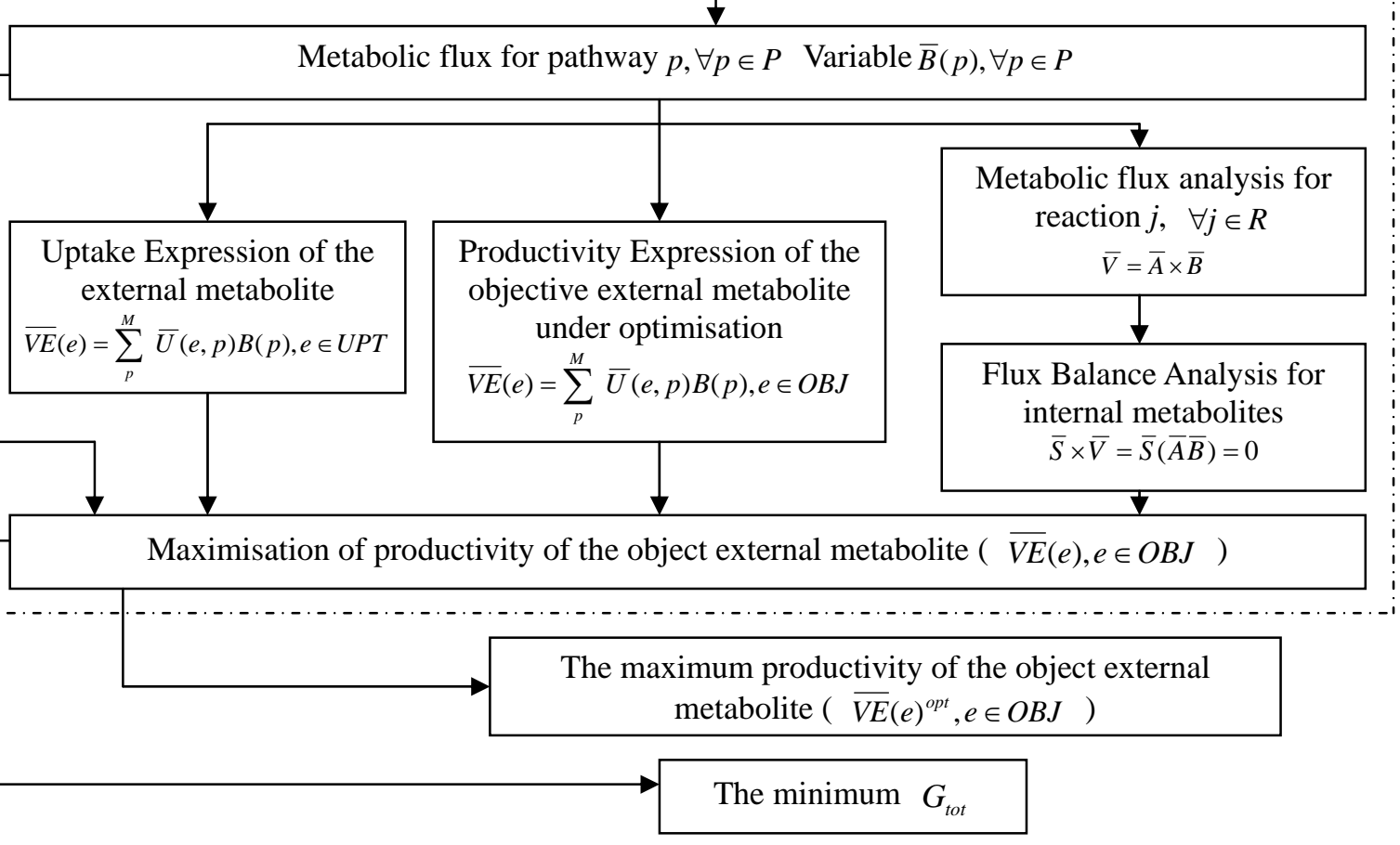

The optimal operating condition $p H=p H^{o p t}, I=I^{o p t}$

Fig. B1 Optimisation Algorithm 


\section{Figures}

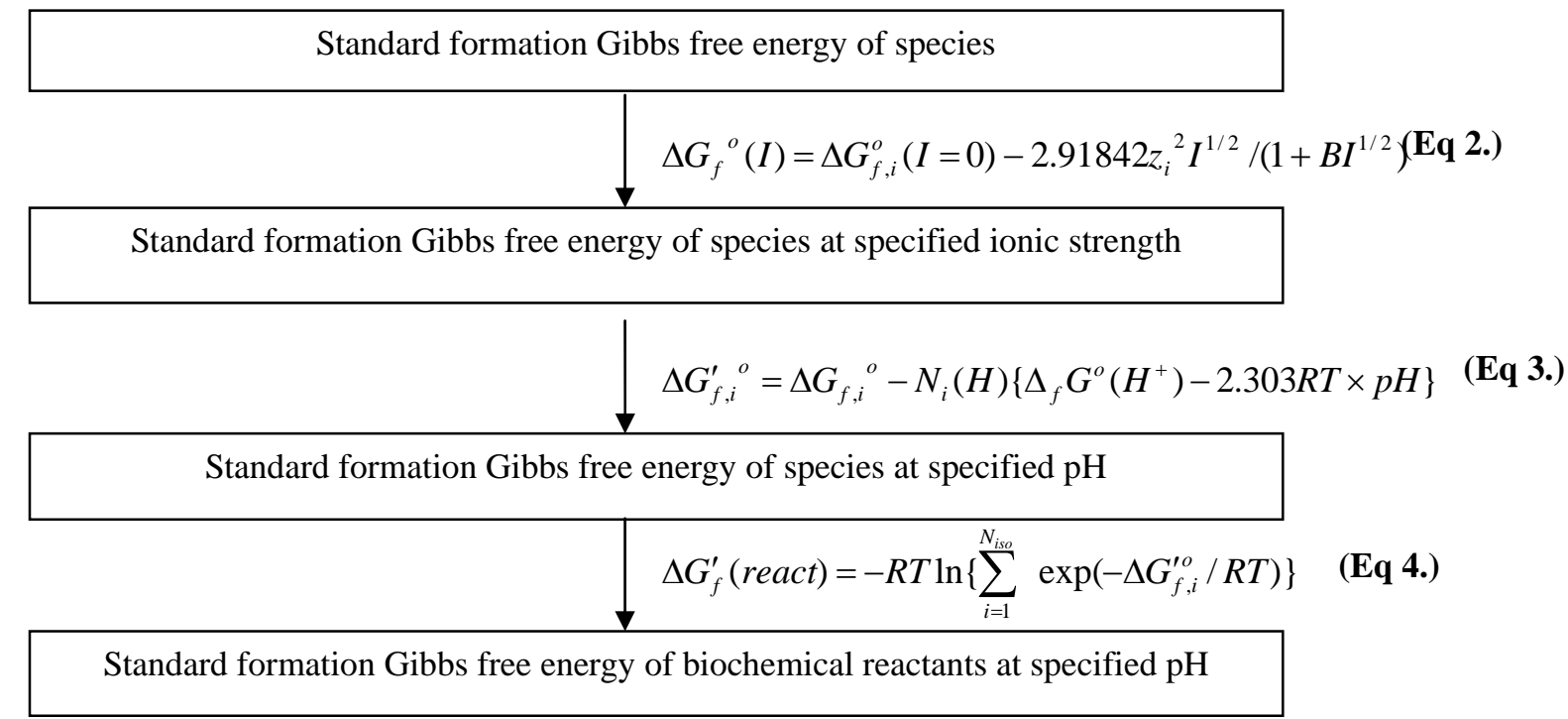

Fig. 1 Calculation of the standard formation Gibbs free energy for biochemical reactants

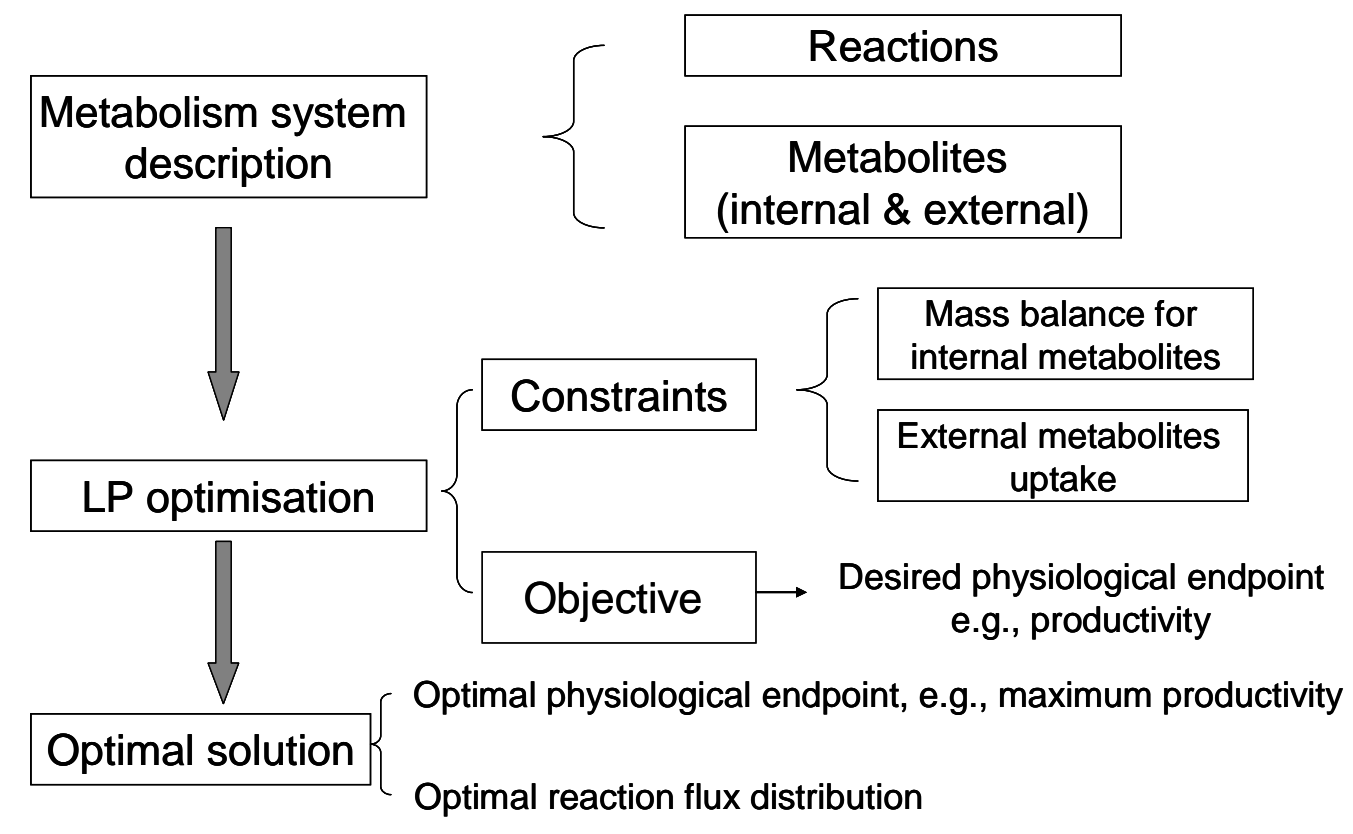

Fig. 2 Flux balance analysis 


\section{Module 1}

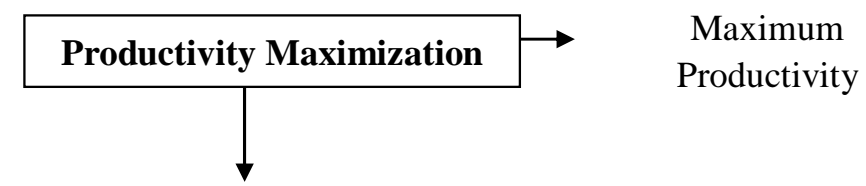

Optimal pathway flux distribution

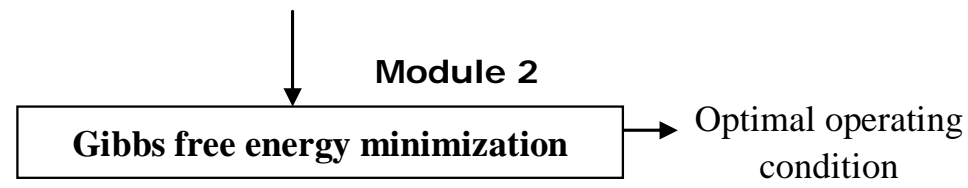

Fig. 3 Modules for Optimisation

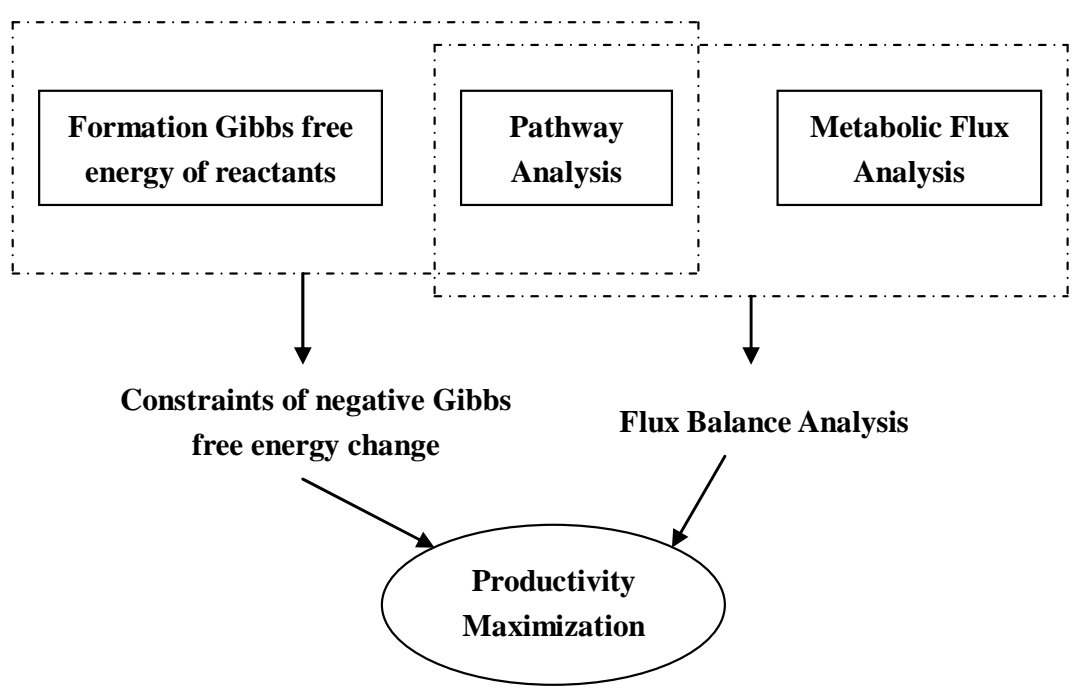

Fig. 4 Module 1: Productivity maximization

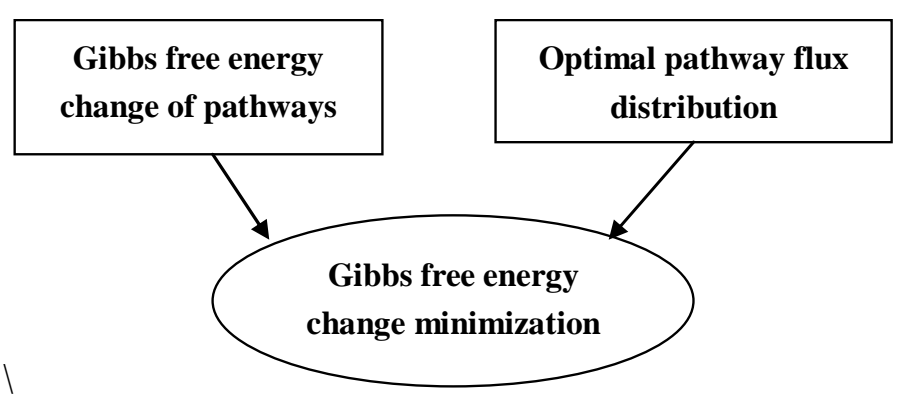

Fig. 5 Module 2: Gibbs free energy minimization 


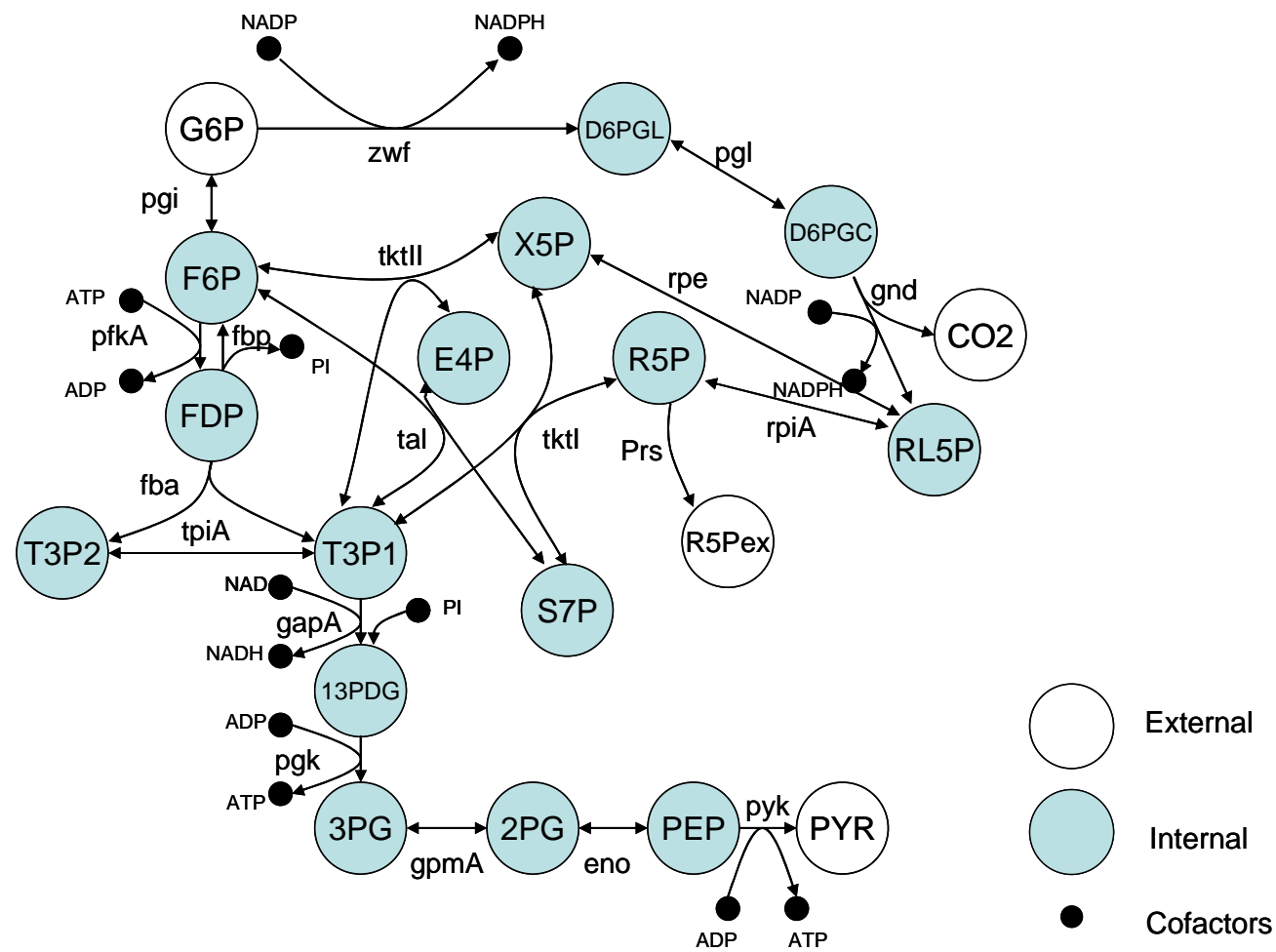

Fig. 6 Overview of the metabolic network of Glycolysis and PPP in E.coli model

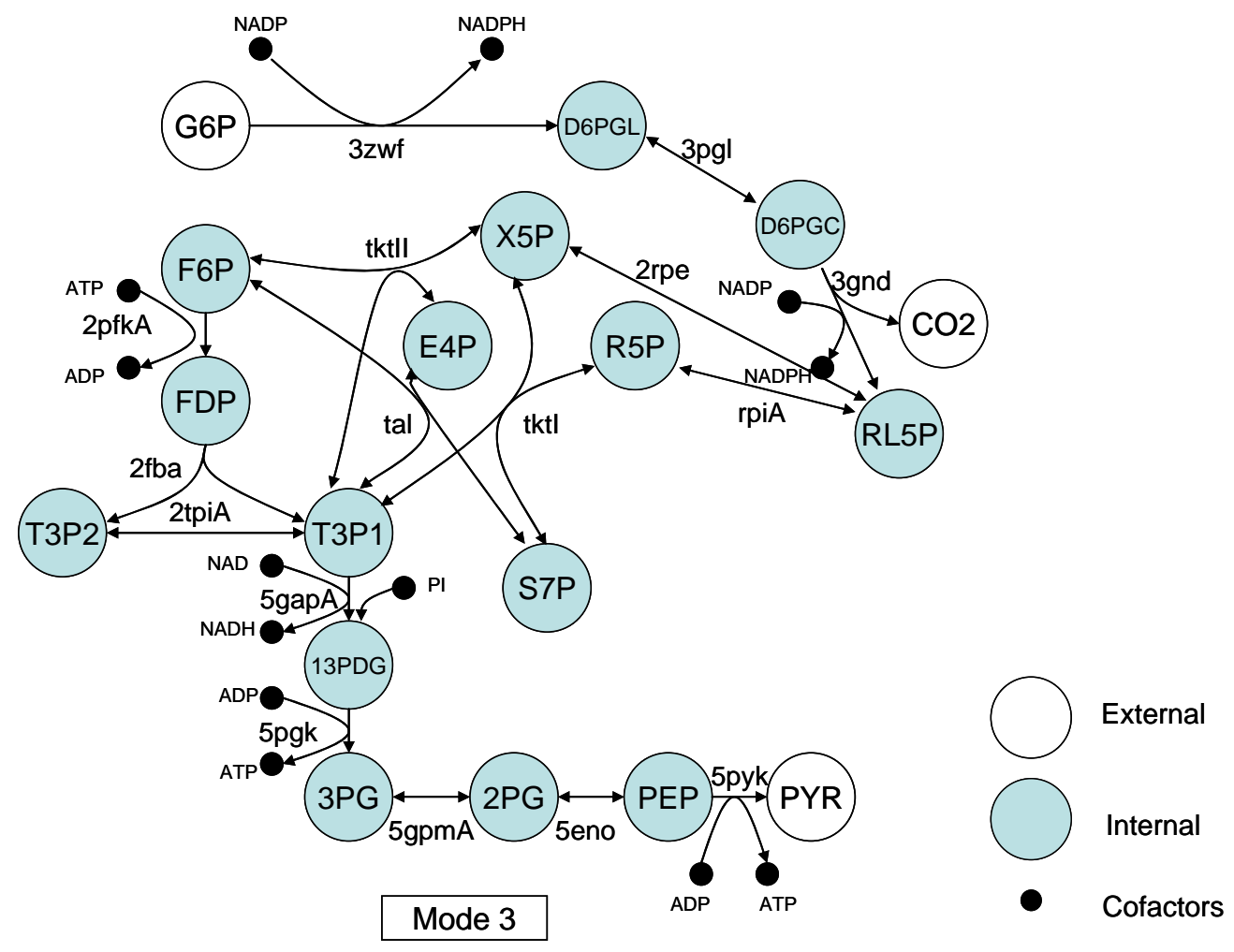

Fig. 7 Graphical representation of the pathway mode 3 pertaining to the reaction scheme 

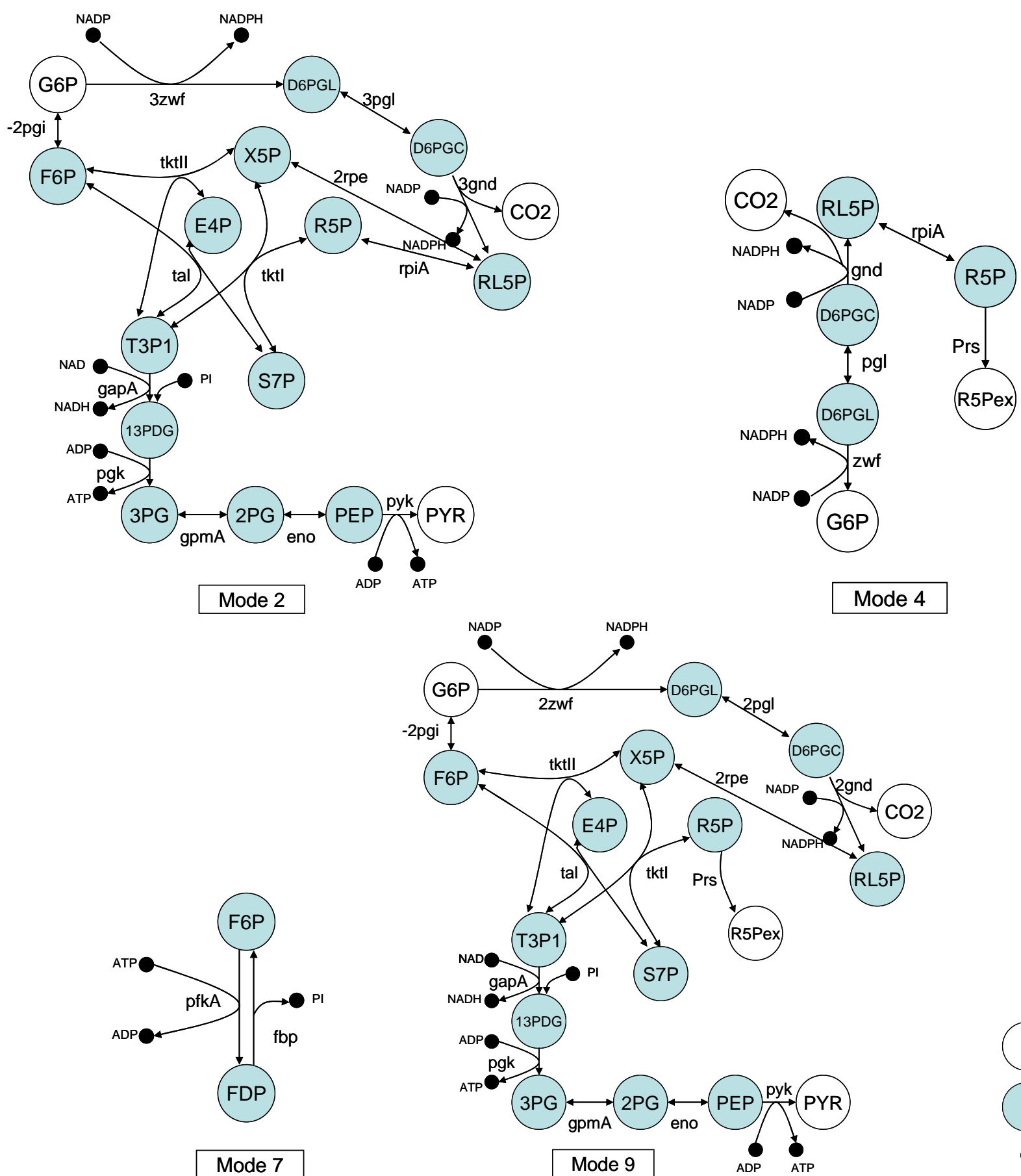

Mode 4

Mode 7

Fig. 8 Graphical representation of the pathway mode 2, 4,7 and 9 


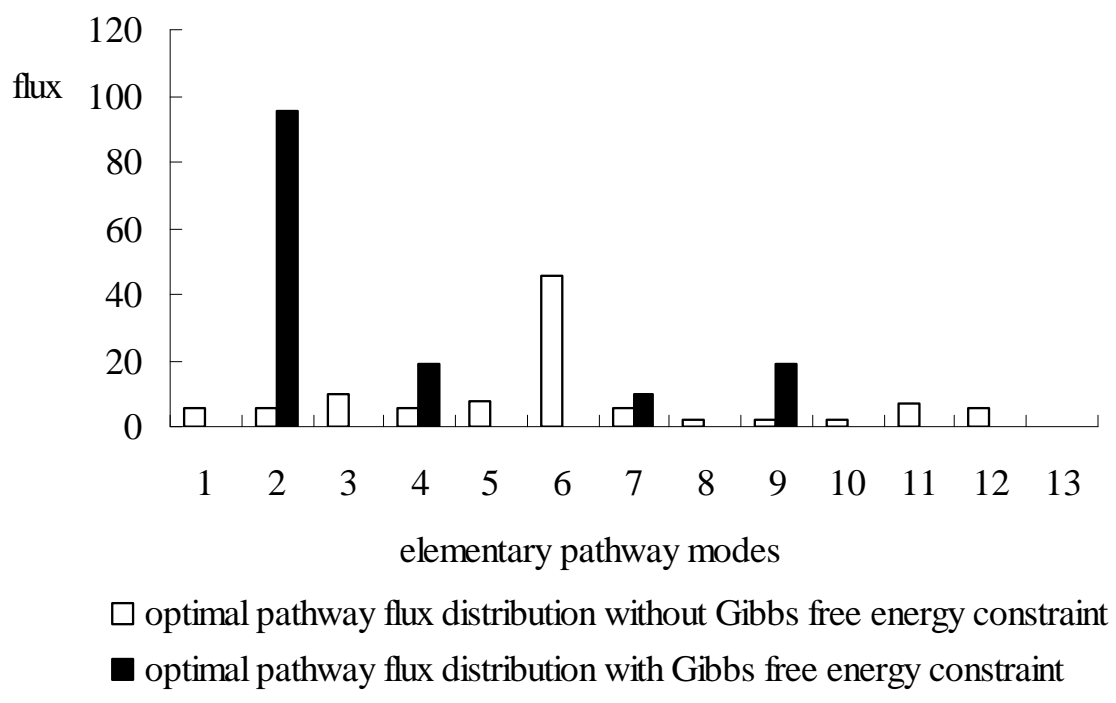

Fig. 9 Comparison of the optimal pathway flux distribution with and without Gibbs free energy constraint

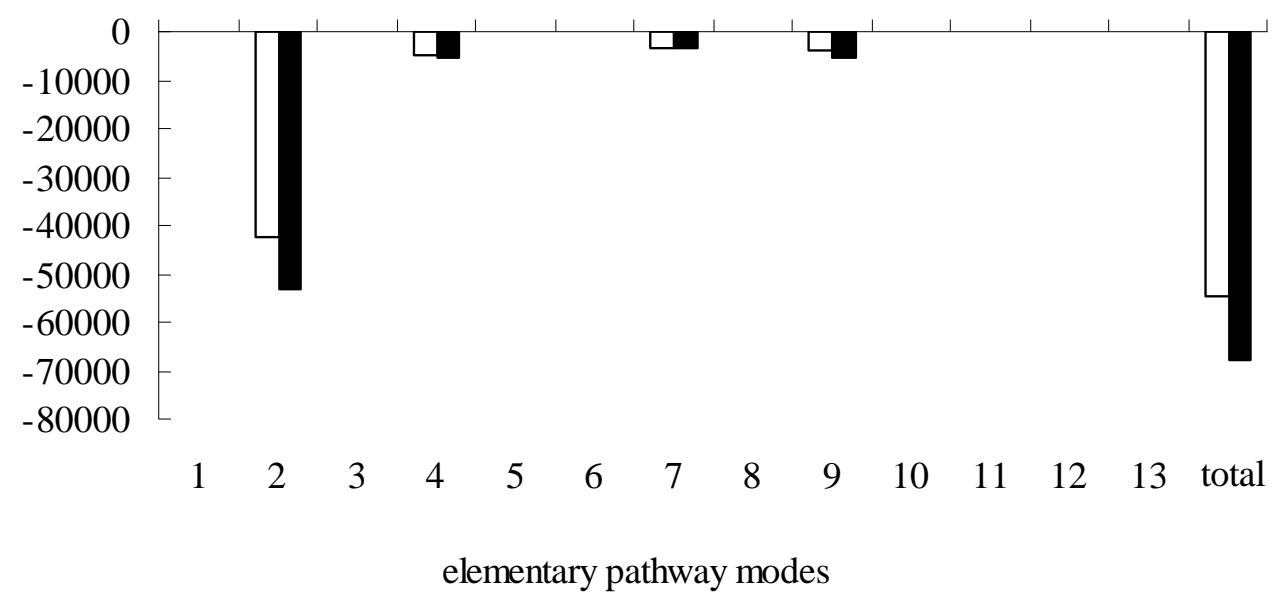

$\square$ Gibbs free energy change for elementary pathways before minimisation

- Gibbs free energy change for elementary pathways after minimisation

Fig. 10 Comparison of the Gibbs free energy change for elementary pathways before and after minimisation 


\section{Tables}

Table 1 Properties for $A T P^{4-}$, $H A T P^{3-}, H_{2} A T P^{2-}$ at $25^{\circ} \mathrm{C}, 1 \mathrm{bar}$, and $\boldsymbol{I}=\mathbf{0}$

\begin{tabular}{llll}
\hline Species & $\Delta G_{f}{ }^{o} / \mathrm{kJ} \cdot \mathrm{mol}^{-1}$ & Charges $z_{i}$ & Hydrogen atom numbers $N_{i}(H)$ \\
\hline$A T P^{4-}$ & -2573.49 & 4 & 12 \\
$H A T P^{3-}$ & -2616.87 & 3 & 13 \\
$H_{2} A T P^{2-}$ & -2643.58 & 2 & 14 \\
\hline
\end{tabular}

Table 2 Metabolic reactions of glycolysis and PPP in E.coli model

\begin{tabular}{llll}
\hline Enzyme & Gene & Rxn no. & Reaction \\
\hline Glycolysis(10) & pgi & 1 & $G 6 P \leftrightarrow F 6 P$ \\
Phosphoglucose isomerase & pfkA & 2 & $F 6 P+A T P \rightarrow A D P+F D P$ \\
Phosphafructokinase & fbp & 3 & $F D P \rightarrow F 6 P+P I$ \\
Fructose-1,6-bisphosphatase & fba & 4 & $F D P \leftrightarrow T 3 P 1+T 3 P 2$ \\
Fructose-1,6-bisphophate aldolase & tpiA & 5 & $T 3 P 1 \leftrightarrow T 3 P 2$ \\
Triosphosphate isomerase & gapA & 6 & $T 3 P 1+P I+N A D \rightarrow N A D H+13 P D G$ \\
Glyceraldehyde-3-phosphate dehydrogenase & pgk & 7 & $13 P D G+A D P \rightarrow A T P+3 P G$ \\
Phosphoglycerate kinase & gpmA & 8 & $3 P G \leftrightarrow 2 P G$ \\
Phosphoglycerate mutes & eno & 9 & $2 P G \leftrightarrow P E P$ \\
Enolase & pyk & 10 & $P E P+A D P \rightarrow A T P+P Y R$ \\
Pyruvate kinase & & & \\
Pentose phosphate pathway (PPP)(9) & zwf & 11 & $G 6 P+N A D P \rightarrow N A D P H+D 6 P G L$ \\
Glucose-6-phosphate dehydrogenase & pgl & 12 & $D 6 P G L \leftrightarrow D 6 P G C$ \\
6-Phophogluconolactonse & gnd & 13 & $D 6 P G C+N A D P \rightarrow N A D P H+C O_{2}+R L 5 P$ \\
6-Phosphogluconate dehydrogenase & rpiA & 14 & $R L 5 P \leftrightarrow R 5 P$ \\
Ribose-5-phosphate isomerase & rpe & 15 & $R L 5 P \leftrightarrow X 5 P$ \\
Ribulose phosphate 3-epimerase & tktI & 16 & $X 5 P+R 5 P \leftrightarrow T 3 P 1+S 7 P$ \\
Transketolase 1 & tal & 17 & $T 3 P 1+S 7 P \leftrightarrow E 4 P+F 6 P$ \\
Transaldolase & TktII & 18 & $X 5 P+E 4 P \leftrightarrow F 6 P+T 3 P 1$ \\
Transketolase 2 & Prs & 19 & $R 5 P \rightarrow R 5 P e x$ \\
5-Phosphoribosyl-1-pyrophosphate synthetase & &
\end{tabular}


Table 3 Metabolites of glycolysis and PPP in E.coli model

\begin{tabular}{|c|c|}
\hline Abbreviation & Compound \\
\hline \multicolumn{2}{|c|}{ External metabolites(4) } \\
\hline$G 6 P$ & Glucose 6-phosphate \\
\hline PYR & Pyruvate \\
\hline $\mathrm{CO}_{2}$ & Carbon dioxide \\
\hline$R 5 P e x$ & Ribose 5-phosphate (external) \\
\hline \multicolumn{2}{|c|}{ Internal metabolites(15) } \\
\hline$F 6 P$ & Fructose 6-phosphate \\
\hline$F D P$ & Fructose 1,6-diphosphate \\
\hline$T 3 P 1$ & Glyceraldehyde-3-phosphate \\
\hline T3P2 & Dihydroxyacetone phosphate \\
\hline $13 P D G$ & $1,3-P$ - $d$ glycerate \\
\hline $3 P G$ & 3-P- $d$ glycerate \\
\hline $2 P G$ & 2-P- $d$ glycerate \\
\hline PEP & Phosphoenolpyruvate \\
\hline D6PGL & $d$-6-Phosphogluconate \\
\hline$D 6 P G C$ & $d$-6-Phosphoglucono- $\delta$-lactone \\
\hline$R L 5 P$ & $d$-Ribulose 5-phosphate \\
\hline$R 5 P$ & Ribose 5-phosphate \\
\hline$X 5 P$ & Xylulose-5-phosphate \\
\hline$S 7 P$ & $d$-Sedoheptulose-7-P \\
\hline$E 4 P$ & Erythrose 4-phosphate \\
\hline \multicolumn{2}{|l|}{ Cofactors(7) } \\
\hline ATP & Adenosine triphosphate \\
\hline$A D P$ & Adenosine diphosphate \\
\hline$N A D$ & \multirow{2}{*}{ Nicotinamide adenine dinucleotide } \\
\hline$N A D H$ & \\
\hline$N A D P$ & \multirow{2}{*}{ Nicotinamide adenine dinucleotide phosphate } \\
\hline$N A D P H$ & \\
\hline$P I$ & Phosphate \\
\hline
\end{tabular}


Table 4 Elementary path modes of the combined glycolysis and PPP system

\begin{tabular}{|c|c|c|}
\hline Mode & 0verall equation & Steps \\
\hline 1 & $\begin{array}{l}G 6 P+3 A D P+2 P i+2 N A D \rightarrow \\
3 A T P+2 N A D H+2 P y r\end{array}$ & $\begin{array}{l}\text { pgi, pfkA, fba, tpiA, 2gapA, 2pgk, 2gpmA, 2eno, } \\
\text { 2pyk }\end{array}$ \\
\hline 2 & $\begin{array}{l}G 6 P+2 A D P+P i+N A D+6 N A D P \rightarrow \\
2 A T P+N A D H+6 N A D H P+3 C O_{2}+P y r\end{array}$ & $\begin{array}{l}\text {-2pgi, gapA, 3zwf, 3pgl, 3gnd, rpiA, 2rpe, tktI, tal, } \\
\text { tktII, pgk, gpmA, eno, pyk, }\end{array}$ \\
\hline 3 & $\begin{array}{l}3 G 6 P+8 A D P+5 P i+5 N A D+6 N A D P \rightarrow \\
8 A T P+5 N A D H+6 N A D H P+3 C O_{2}+5 P y r\end{array}$ & $\begin{array}{l}\text { 2pfkA, 2fba, 2tpiA, 5gapA, 3zwf, 3pgl, 3gnd, rpiA, } \\
\text { 2rpe, tktI, tal, tktII, 5pgk, 5gpmA, 5eno, pyk }\end{array}$ \\
\hline 4 & $G 6 P+2 \mathrm{NADP} \rightarrow 2 \mathrm{NADPH}+\mathrm{CO}_{2}+\mathrm{R} 5 \mathrm{Pex}$ & zwf, pgl, gnd, rpiA, Prs \\
\hline 5 & $5 G 6 P+A T P \rightarrow A D P+6 R 5 P e x$ & $\begin{array}{l}\text { 5pgi, pfkA, fba, tpiA, 4rpiA, -4rpe, -2tktI, -2tal, } \\
\text {-2tktII, 6Prs }\end{array}$ \\
\hline 6 & $G 6 P+12 N A D P \rightarrow 12 N A D P H+P i+6 C_{2}$ & $\begin{array}{l}\text {-5pgi, -fba, -tpiA, 6zwf, 6pgl, 6gnd, 2rpiA, 4rpe, } \\
\text { 2tktI, 2tal, 2tktII, fbp }\end{array}$ \\
\hline 7 & $A T P \rightarrow A D P+P i$ & pfk, fbp \\
\hline 8 & $\begin{array}{l}2 A D P+P i+N A D+3 R 5 P e x \rightarrow \\
2 A T P+N A D H+2 G 6 P+P y r\end{array}$ & $\begin{array}{l}\text {-2pgi, gapA, -2rpiA, 2rpe, tktI, tal, tktII, pgk, gpmA, } \\
\text { eno, pyk, -3Prs }\end{array}$ \\
\hline 9 & $\begin{array}{l}2 A D P+P i+N A D+4 N A D P+R 5 P e x \rightarrow \\
2 C O_{2}+2 A T P+N A D H+4 N A D H P+P y r\end{array}$ & $\begin{array}{l}\text {-2pgi, gapA, 2zwf, 2pgl, 2gnd, 2rpe, tktI, tal, tktII, } \\
\text { pgk, gpmA, eno, pyk, -Prs }\end{array}$ \\
\hline 10 & $\begin{array}{l}8 A D P+5 P i+5 N A D+3 R 5 P e x \rightarrow \\
8 A T P+5 N A D H+5 P y r\end{array}$ & $\begin{array}{l}\text { 2pfkA, 2fba, 2tpiA, 5gapA, -2rpiA, 2rpe, tktI, tal, } \\
\text { tktII, 5pgk, 5gpmA, 5eno, 5pyk, -3Prs }\end{array}$ \\
\hline 11 & $\begin{array}{l}8 A D P+5 P i+5 N A D+4 N A D P+2 G 6 P+R 5 P e x \rightarrow \\
2 C O_{2}+8 A T P+5 N A D H+4 N A D H P+5 P y r\end{array}$ & $\begin{array}{l}\text { 2pfkA, 2fba, 2tpiA, 5gapA, 2zwf, 2pgl, 2gnd, 2rpe, } \\
\text { tktI, tal, tktII, 5pgk, 5gpmA, 5eno, 5pyk, -Prs }\end{array}$ \\
\hline 12 & $6 R 5 P e x \rightarrow 5 G 6 P+P i$ & $\begin{array}{l}\text {-5pgi, fbp, -fba, -tpiA, -4rpiA, 4rpe, 2tktI, 2tal, } \\
\text { 2tktII,-6 Prs }\end{array}$ \\
\hline 13 & $8 N A D P+2 R 5 P e x \rightarrow 4 C O_{2}+8 N A D P H+G 6 P+P i$ & $\begin{array}{l}\text {-5pgi, fbp, -fba, -tpiA, 4zwf, 4pgl, 4gnd, 4rpe, 2tktI, } \\
\text { 2tal, 2tktII, -Prs }\end{array}$ \\
\hline
\end{tabular}


Table 5 Stoichiometric matrix $\bar{S}_{(i, j)}$ in terms of internal metabolites $i \in I M \quad$ in reactions $j \in R$

\begin{tabular}{|c|c|c|c|c|c|c|c|c|c|c|c|c|c|c|c|}
\hline \multirow{2}{*}{ reactions } & \multicolumn{15}{|c|}{ internal metabolites } \\
\hline & $F 6 P$ & $F D P$ & T3P1 & $T 3 P 2$ & $13 P D G$ & $3 P G$ & $2 P G$ & PEP & $D 6 P G L$ & $D 6 P G C$ & $R L 5 P$ & $R 5 P$ & $X 5 P$ & $S 7 P$ & $E 4 P$ \\
\hline 1 & 1 & 0 & 0 & 0 & 0 & 0 & 0 & 0 & 0 & 0 & 0 & 0 & 0 & 0 & 0 \\
\hline 2 & -1 & 1 & 0 & 0 & 0 & 0 & 0 & 0 & 0 & 0 & 0 & 0 & 0 & 0 & 0 \\
\hline 3 & 1 & -1 & 0 & 0 & 0 & 0 & 0 & 0 & 0 & 0 & 0 & 0 & 0 & 0 & 0 \\
\hline 4 & 0 & -1 & 1 & 1 & 0 & 0 & 0 & 0 & 0 & 0 & 0 & 0 & 0 & 0 & 0 \\
\hline 5 & 0 & 0 & -1 & 1 & 0 & 0 & 0 & 0 & 0 & 0 & 0 & 0 & 0 & 0 & 0 \\
\hline 6 & 0 & 0 & -1 & 0 & 1 & 0 & 0 & 0 & 0 & 0 & 0 & 0 & 0 & 0 & 0 \\
\hline 7 & 0 & 0 & 0 & 0 & -1 & 1 & 0 & 0 & 0 & 0 & 0 & 0 & 0 & 0 & 0 \\
\hline 8 & 0 & 0 & 0 & 0 & 0 & -1 & 1 & 0 & 0 & 0 & 0 & 0 & 0 & 0 & 0 \\
\hline 9 & 0 & 0 & 0 & 0 & 0 & 0 & -1 & 1 & 0 & 0 & 0 & 0 & 0 & 0 & 0 \\
\hline 10 & 0 & 0 & 0 & 0 & 0 & 0 & 0 & -1 & 0 & 0 & 0 & 0 & 0 & 0 & 0 \\
\hline 11 & 0 & 0 & 0 & 0 & 0 & 0 & 0 & 0 & 1 & 0 & 0 & 0 & 0 & 0 & 0 \\
\hline 12 & 0 & 0 & 0 & 0 & 0 & 0 & 0 & 0 & -1 & 1 & 0 & 0 & 0 & 0 & 0 \\
\hline 13 & 0 & 0 & 0 & 0 & 0 & 0 & 0 & 0 & 0 & -1 & 1 & 0 & 0 & 0 & 0 \\
\hline 14 & 0 & 0 & 0 & 0 & 0 & 0 & 0 & 0 & 0 & 0 & -1 & 1 & 0 & 0 & 0 \\
\hline 15 & 0 & 0 & 0 & 0 & 0 & 0 & 0 & 0 & 0 & 0 & -1 & 0 & 1 & 0 & 0 \\
\hline 16 & 0 & 0 & 1 & 0 & 0 & 0 & 0 & 0 & 0 & 0 & 0 & -1 & -1 & 1 & 0 \\
\hline 17 & 1 & 0 & -1 & 0 & 0 & 0 & 0 & 0 & 0 & 0 & 0 & 0 & 0 & -1 & 1 \\
\hline 18 & 1 & 0 & 1 & 0 & 0 & 0 & 0 & 0 & 0 & 0 & 0 & 0 & -1 & 0 & -1 \\
\hline 19 & 0 & 0 & 0 & 0 & 0 & 0 & 0 & 0 & 0 & 0 & 0 & -1 & 0 & 0 & 0 \\
\hline
\end{tabular}

Table 6 Stoichiometric matrix $\bar{A}_{(j, p)}$ in terms of reactions $j \in R$ in elementary pathways $p \in P$

\begin{tabular}{|c|c|c|c|c|c|c|c|c|c|c|c|c|c|c|c|c|c|c|c|}
\hline \multirow{2}{*}{ Elementary pathways } & \multicolumn{19}{|c|}{ Reactions } \\
\hline & 1 & 2 & 3 & 4 & 5 & 6 & 7 & 8 & 9 & 10 & 11 & 12 & 13 & 14 & 15 & 16 & 17 & 18 & 19 \\
\hline 1 & 1 & 1 & 0 & 1 & 1 & 2 & 2 & 2 & 2 & 2 & 0 & 0 & 0 & 0 & 0 & 0 & 0 & 0 & 0 \\
\hline 2 & -2 & 0 & 0 & 0 & 0 & 1 & 1 & 1 & 1 & 1 & 3 & 3 & 3 & 1 & 2 & 1 & 1 & 1 & 0 \\
\hline 3 & 0 & 2 & 0 & 2 & 2 & 5 & 5 & 5 & 5 & 5 & 3 & 3 & 3 & 1 & 2 & 1 & 1 & 1 & 0 \\
\hline 4 & 0 & 0 & 0 & 0 & 0 & 0 & 0 & 0 & 0 & 0 & 1 & 1 & 1 & 1 & 0 & 0 & 0 & 0 & 1 \\
\hline 5 & 5 & 1 & 0 & 1 & 1 & 0 & 0 & 0 & 0 & 0 & 0 & 0 & 0 & 4 & -4 & -2 & -2 & -2 & 6 \\
\hline 6 & -5 & 0 & 1 & -1 & -1 & 0 & 0 & 0 & 0 & 0 & 6 & 6 & 6 & 2 & 4 & 2 & 2 & 2 & 0 \\
\hline 7 & 0 & 1 & 1 & 0 & 0 & 0 & 0 & 0 & 0 & 0 & 0 & 0 & 0 & 0 & 0 & 0 & 0 & 0 & 0 \\
\hline 8 & -2 & 0 & 0 & 0 & 0 & 1 & 1 & 1 & 1 & 1 & 0 & 0 & 0 & -2 & 2 & 1 & 1 & 1 & -3 \\
\hline 9 & -2 & 0 & 0 & 0 & 0 & 1 & 1 & 1 & 1 & 1 & 2 & 2 & 2 & 0 & 2 & 1 & 1 & 1 & -1 \\
\hline 10 & 0 & 2 & 0 & 2 & 2 & 5 & 5 & 5 & 5 & 5 & 0 & 0 & 0 & -2 & 2 & 1 & 1 & 1 & -3 \\
\hline 11 & 0 & 2 & 0 & 2 & 2 & 5 & 5 & 5 & 5 & 5 & 2 & 2 & 2 & 0 & 2 & 1 & 1 & 1 & -1 \\
\hline 12 & -5 & 0 & 1 & -1 & -1 & 0 & 0 & 0 & 0 & 0 & 0 & 0 & 0 & -4 & 4 & 2 & 2 & 2 & -6 \\
\hline 13 & -5 & 0 & 1 & -1 & -1 & 0 & 0 & 0 & 0 & 0 & 4 & 4 & 4 & 0 & 4 & 2 & 2 & 2 & -1 \\
\hline
\end{tabular}


Table 7 Stoichiometric matrix $\bar{U}_{(p, e)}$ in terms of external metabolites and cofactors $e \in E M$ in elementary pathways $p \in P$

\begin{tabular}{|c|ccccccccccccc|}
\hline \multirow{2}{*}{ External metabolites and cofactors } & \multicolumn{110}{|c|}{ Elementary pathways } \\
\cline { 2 - 14 } & 1 & 2 & 3 & 4 & 5 & 6 & 7 & 8 & 9 & 10 & 11 & 12 & 13 \\
\hline$A T P$ & 3 & 2 & 8 & 0 & -1 & 0 & -1 & 2 & 2 & 8 & 8 & 0 & 0 \\
$A D P$ & -3 & -2 & -8 & 0 & 1 & 0 & 1 & -2 & -2 & -8 & -8 & 0 & 0 \\
$G 6 P$ & -1 & -1 & -3 & -1 & -5 & -1 & 0 & 2 & 0 & 0 & -2 & 5 & 1 \\
$P Y R$ & 2 & 1 & 5 & 0 & 0 & 0 & 0 & 1 & 1 & 5 & 5 & 0 & 0 \\
$R 5 P e x$ & 0 & 0 & 0 & 1 & 6 & 0 & 0 & -3 & -1 & -3 & -1 & -6 & -2 \\
$C O_{2}$ & 0 & 3 & 3 & 1 & 0 & 6 & 0 & 0 & 2 & 0 & 2 & 0 & 4 \\
$N A D$ & -2 & -1 & -5 & 0 & 0 & 0 & 0 & -1 & -1 & -5 & -5 & 0 & 0 \\
$N A D H$ & 2 & 1 & 5 & 0 & 0 & 0 & 0 & 1 & 1 & 5 & 5 & 0 & 0 \\
$N A D P$ & 0 & -6 & -6 & -2 & 0 & -12 & 0 & 0 & -4 & 0 & -4 & 0 & -8 \\
$N A D P H$ & 0 & 6 & 6 & 2 & 0 & 12 & 0 & 0 & 4 & 0 & 4 & 0 & 8 \\
$P I$ & -2 & -1 & -5 & 0 & 0 & 1 & 1 & -1 & -1 & -5 & -5 & 1 & 1 \\
\hline
\end{tabular}

Table 8 Standard formation Gibbs free energy, charges and hydrogen atom numbers for species

\begin{tabular}{lccc}
\hline Species & $\Delta G_{f}{ }^{o} / \mathrm{kJ} \cdot \mathrm{mol}^{-1}$ & Charges $\mathrm{Z}_{i}$ & Hydrogen atom numbers $N_{i}(H)$ \\
\hline$G 6 P^{2-}$ & -1763.94 & 2 & 11 \\
$P Y R^{-}$ & -472.27 & 1 & 3 \\
$R 5 P^{2-}$ & -1605.34 & 2 & 9 \\
$N A D^{-}$ & 0 & 1 & 26 \\
$N A D H^{2-}$ & 22.65 & 2 & 26 \\
$N A D P^{3-}$ & 0 & 3 & 25 \\
$N A D P H^{4-}$ & 25.99 & 4 & 25 \\
$H P O_{4}{ }^{2-}$ & -1095.1 & 2 & 1 \\
$H_{2} P_{4}{ }^{-}$ & -1137.3 & 1 & 2 \\
$A T P^{4-}$ & -2573.49 & 4 & 12 \\
$H A T P^{3-}$ & -2616.87 & 3 & 13 \\
$H_{2} A T P^{2-}$ & -2643.58 & 2 & 14 \\
$A D P^{3-}$ & -1711.55 & 3 & 12 \\
$H A D P^{2-}$ & -1752.53 & 2 & 13 \\
$H_{2} A D P^{-}$ & -1777.42 & 1 & 14 \\
\hline
\end{tabular}


Table 9 Standard formation Gibbs free energy for external metabolites and cofactors at $p H=6.8$, $I=0.28$

\begin{tabular}{lc}
\hline External metabolites and cofactors & $\Delta G_{f}^{\prime o} / \mathrm{kJ} \cdot \mathrm{mol}^{-1}$ \\
\hline$G 6 P$ & -1331.29 \\
PYR & -354.198 \\
R5Pex & -1251.96 \\
NAD & 1029.701 \\
NADH & 1049.842 \\
NADP & 983.3745 \\
NADPH & 1003.511 \\
PI & -1230.57 \\
ATP & -2376.47 \\
ADP & -1508.15 \\
\hline
\end{tabular}

Table 10 Comparison of optimal pathway flux distribution and their corresponding Gibbs free energy changes

\begin{tabular}{c|cc|cc}
\hline $\begin{array}{c}\text { Elementary } \\
\text { Modes }\end{array}$ & $\begin{array}{c}\text { without } \Delta G \text { constraint } \\
\text { Pathway flux distribution } B(p) \\
(\mathrm{mmol} / \mathrm{g} D C W \mathrm{C})\end{array}$ & $\begin{array}{c}\Delta G \\
\mathrm{~kJ} / \mathrm{mol}\end{array}$ & $\begin{array}{c}\text { Pathway flux distribution } B(p) \\
(\mathrm{mmol} / \mathrm{g} D C W h)\end{array}$ & $\begin{array}{c}\Delta G \\
\mathrm{~kJ} / \mathrm{mol}\end{array}$ \\
\hline 1 & 5.62 & 3390.493 & 0 & 0 \\
2 & 5.62 & -2495.21 & 95.746 & -42509.4 \\
3 & 10 & 7625.8 & 0 & 0 \\
4 & 5.62 & -1375.01 & 19.254 & -4710.65 \\
5 & 7.976 & 185.676 & 0 & 0 \\
6 & 45.785 & -85583.7 & 0 & 0 \\
7 & 5.62 & -2124.45 & 9.627 & -3639.07 \\
8 & 1.808 & 524.388 & 0 & 0 \\
9 & 1.808 & -360.417 & 19.254 & -3837.68 \\
10 & 1.808 & 2706.131 & 0 & 0 \\
11 & 7.096 & 7147.792 & 0 & 0 \\
12 & 5.62 & -2255.29 & 0 & 0 \\
13 & 0 & 0 & 0 & 0 \\
\hline
\end{tabular}


Table 11 Reaction flux distribution derived from FBA alone

\begin{tabular}{c|c}
\hline Reaction & $\begin{array}{c}\text { Reaction flux distribution } V(j) \\
(\mathrm{mmol} / \mathrm{gDCWh})\end{array}$ \\
\hline 1 & -230 \\
2 & 46 \\
3 & 46 \\
4 & 0 \\
5 & 0 \\
6 & 115 \\
7 & 115 \\
8 & 115 \\
9 & 115 \\
10 & 115 \\
11 & 345 \\
12 & 345 \\
13 & 345 \\
14 & 115 \\
15 & 230 \\
16 & 115 \\
17 & 115 \\
18 & 115 \\
19 & 0 \\
\hline
\end{tabular}

Table 12 Pathway analysis based on the result of reaction flux distribution derived from FBA alone

\begin{tabular}{c|c|c}
\hline Pathway & $\begin{array}{c}\text { Reaction flux distribution } B(p) \\
(\text { mmol/gDCWh })\end{array}$ & $\begin{array}{c}\Delta G \\
k J / m o l\end{array}$ \\
\hline 1 & 0 & 0 \\
2 & 0 & 0 \\
3 & 23 & 17539.34 \\
4 & 0 & 0 \\
5 & 0 & 0 \\
6 & 46 & -85985.5 \\
7 & 0 & 0 \\
8 & 0 & 0 \\
9 & 0 & 0 \\
10 & 0 & 0 \\
11 & 0 & 0 \\
12 & 0 & 0 \\
13 & 0 & 0 \\
\hline
\end{tabular}


Table 13 Standard formation Gibbs free energy at optimal condition

\begin{tabular}{ll}
\hline External metabolites and cofactors & $\Delta G_{f}^{\text {opt }} / \mathrm{kJ} \cdot \mathrm{mol}^{-1}$ \\
\hline$G 6 P$ & -1318.634 \\
$P Y R$ & -350.745 \\
$R 5 P e x$ & -1241.617 \\
$N A D$ & 1059.736 \\
$N A D H$ & 1079.833 \\
$N A D P$ & 1012.137 \\
$N A D P H$ & 1032.171 \\
$P I$ & -1228.872 \\
$A T P$ & -2361.616 \\
$A D P$ & -1493.225 \\
\hline$\Delta G_{\text {tot }}^{\text {opt }} / \mathrm{kJ} \cdot \mathrm{mol}^{-1}$ & -5577.197 \\
\hline
\end{tabular}




\section{Literature Cited}

(1) Nolan, R.P.; Fenley, A.P.; Lee, K. Identification of distributed metabolic objectives in the hypermetabolic liver by flux and energy balance analysis. Metab. Eng. 2006, 8, 30.

(2) Burgard, A.P.; Maranas, C.D. Optimisation-based framework for inferring and testing hypothesized metabolic objective functions. Biotechnol. Bioeng. 2003, 82, 670.

(3) Bonarius, H.P.J.; Schmid, G.; Tramper, J. Flux analysis of underdetermined metabolic networks: the quest for the missing constraints. Trends. Biochem. Sci. 1997, 15, 308.

(4) Edwards, J.S.; Palsson. How will bioinformatics influence metabolic engineering? Biotechnol. Bioeng. 1998, 58, 162.

(5) Varma, A.; Palsson. Metabolic flux balancing: basic concepts, scientific and practical use. Nat. Biotechnol. 1994, 12, 994.

(6) Edwards, J.S.; Ramakrishna, R.; Schilling, C.S.; Palsson. Metabolic Engineering; Marcel Dekker: New York. 1999.

(7) Sauer, U.; Cameron, D.C.; Bailey, J.E. Metabolic capacity of Bacillus subtilis for the production of purine nucleosides, riboflavin, and folic acid. Biotechnol. Bioeng. 1998, 59, 227.

(8) Schilling, C.H.; Edwards, J.S.; Palsson. Towards metabolic phenomics: analysis of genomic data using fluxbalances. Biotechnol. Progr. 1999, 15, 288.

(9) Mahadevan, R.; Edwards, J. S.; Doyle, F. J. III. Dynamic Flux Balance Analysis of Diauxic Growth in Escherichia coli. Biophys. J. 2002, 83, 1331.

(10) Edwards, J.S.; Covert, M.; Palsson. Metabolic modelling of microbes: the flux-balance approach. Environ. Microbiol. 2002, 4, 133.

(11) Papin, J.A.; Price, N.D.; Edwards, J.S.; Palsson. The genome-scale metabolic extreme pathway structure in Haemophilus influenzae shows significant network redundancy. J. Theor. Biol. 2002, 215, 67.

(12) Schilling, C.H.; Letscher, D.; Palsson, B. Theory for the systemic definition of metabolic pathways and their use in interpreting metabolic function from a pathway-oriented perspective. J. Theor. Biol. 2000, 203, 229.

(13) Schuster, S.; Dandekar, T.; Fell, D.A. Detection of elementary flux modes in biochemical networks: a promising tool for analysis and metabolic engineering. Trends Biotechnol. 1999, 17, 53.

(14) Von Stockar, U.; van der Wielen, L.A.M. Back to basics: thermodynamics in biochemical engineering. Adv. Biochem. Eng ./ Biotechnol. 2005, 80, 1.

(15) Mavrovouniotis, M.L. Group contributions for estimating standard Gibbs energies of formation of biochemical compounds in aqueous solution, Biotechnol. Bioeng. 1990, 36, 1070.

(16) Stryer, L. Biochemistry; W.H. Freeman: New York, 1995.

(17) Alberty, R.A. Review: biochemical thermodynamics. Biochemica. et Biophysica. Act. 1994, 1207, 1.

(18) Xu, M.; Smith, R.; Sadhukhan, J. An Optimisation Approach for Productivity and Thermodynamic Performance of Metabolic Systems. AIChE Annual Meeting, 2006.

(19) Wagman, D.D. The NBS Table of chemical thermodynamic properties, J. Phys. Chem. 1982, Ref. Data 11, Supplement No.2.

(20) Alberty, R.A.; Goldberg, R.N. Biochem. J. 1992, 31, 10610.

(21) Clarke, E.C.; Glew, D.N. Evaluation of thermodynamic functions from equilibrium constants. Transactions of the Faraday Society. 1966, 62, 539.

(22) Lee S.Y.; Papoutsakis E.T. (Eds.). Metabolic Engineering; Marcel Dekker: New York, 1999.

(23) Schuster, S.; Fell, D.A.; Dandekar, T. A general definition of metabolic pathways useful for systematic organization and analysis of complex metabolic networks. Nat. Biotechnol. 2000, 18, 326.

(24) Schmidt, K.; Nielsen, J.; Villadsen, J. Quantitative analysis of metabolic fluxes in Escherichia coli, using two-dimensional NMR spectroscopy and complete isotopomer models. J. Biotechnol. 1999, 71, 175.

\section{Nomenclature}




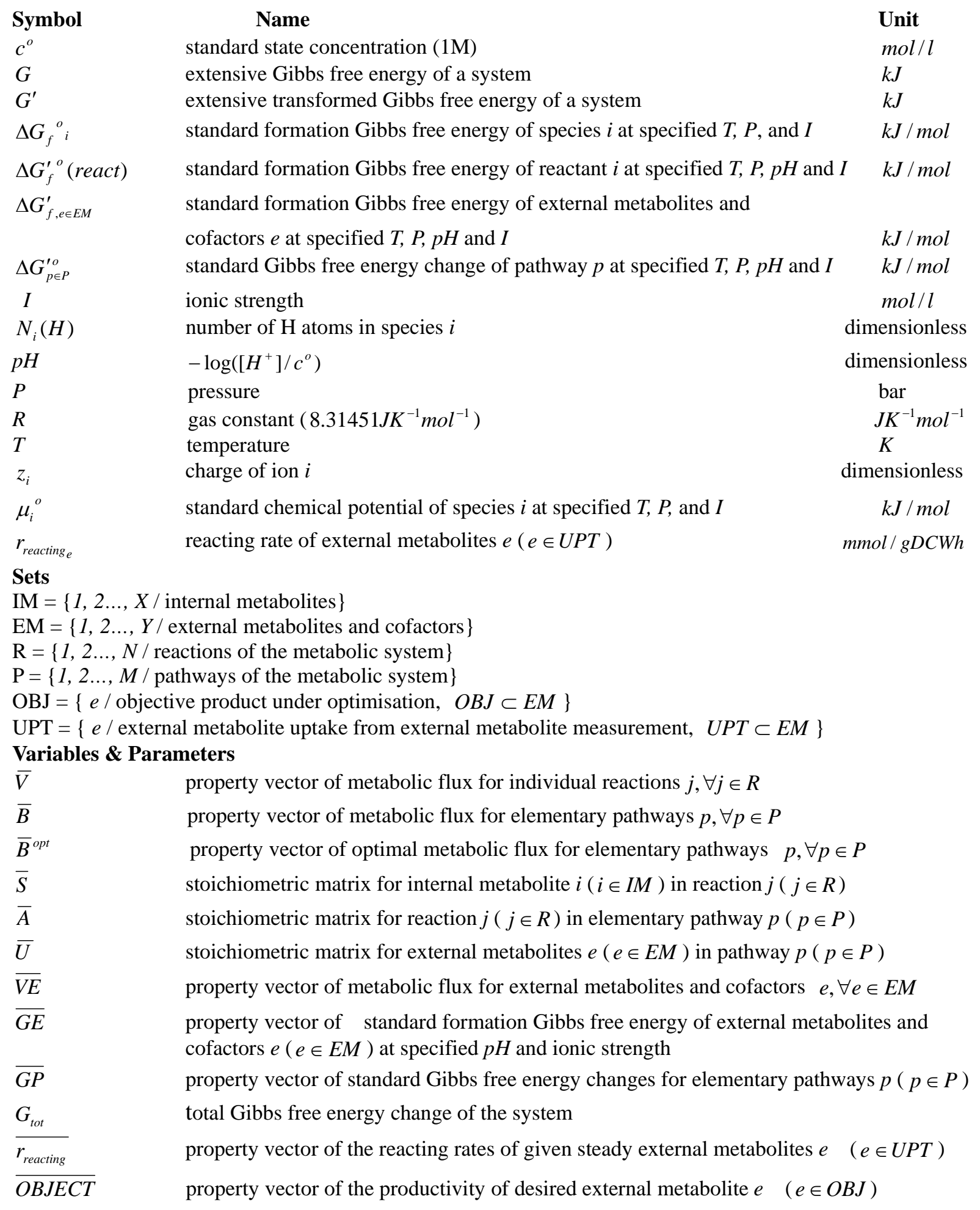

